Ubiquinone and Francisella pathogenicity

\title{
The biosynthetic pathway of ubiquinone contributes to pathogenicity of Francisella
}

\author{
Running title: Ubiquinone and Francisella pathogenicity
}

Katayoun Kazemzadeh ${ }^{1}$, Mahmoud Hajj Chehade ${ }^{1}$, Gautier Hourdoir ${ }^{1}$, Camille Brunet ${ }^{1,2}$, Yvan Caspar $^{2,3}$, Laurent Loiseau ${ }^{4}$, Frederic Barras ${ }^{5,6}$, Fabien Pierrel $^{1}$, and Ludovic Pelosi ${ }^{1 *}$

From ${ }^{1}$ CNRS, CHU Grenoble Alpes, Grenoble INP, TIMC, Université Grenoble Alpes, Grenoble, France; ${ }^{2}$ Laboratoire de Bactériologie-Hygiène Hospitalière, Centre National de Référence des Francisella, Centre Hospitalier Universitaire Grenoble Alpes, 38000, Grenoble, France; ${ }^{3}$ Univ. Grenoble Alpes, CHU Grenoble Alpes, CEA, CNRS, IBS, F-38000 Grenoble, France; ${ }^{4}$ Aix Marseille Université, CNRS, Laboratoire Chimie Bactérienne, Institut Microbiologie de la Méditerranée, 31 Chemin Joseph Aiguier, Marseille 13009, France; ${ }^{5}$ SAMe Unit, Department of Microbiology, Institut Pasteur, Paris, France and ${ }^{6}$ IMM-UMR 2001 CNRS-Institut Pasteur, Paris, France.

*To whom correspondence should be addressed to ludovic.pelosi@univ-grenoble-alpes.fr

Keywords: ubiquinone biosynthesis, coenzyme Q, quinone, aerobic respiration, Francisella tularensis, Francisella novicida.

\section{Abstract}

Francisella tularensis is the causative agent of tularemia. Because of its extreme infectivity and high mortality rate, this pathogen was classified as a biothreat agent. Francisella spp are strict aerobe and ubiquinone (UQ) has been previously identified in these bacteria. While the UQ biosynthetic pathways were extensively studied in Escherichia coli allowing the identification of fifteen Ubi-proteins to date, little is known about Francisella spp. In this study, and using Francisella novicida as a surrogate organism, we first identified $\mathrm{UQ}_{8}$ as the major quinone found in the membranes of this bacterium. Then, we characterized the UQ biosynthetic pathway in F. novicida using a combination of bioinformatics, genetics and biochemical approaches. Our analysis disclosed the presence in Francisella of ten putative Ubi-proteins and we confirmed eight of them by heterologous complementation in $E$. coli. The UQ biosynthetic pathways from F. novicida and E. coli share a similar pattern. 
However, differences were highlighted: the decarboxylase remains unidentified in Francisella spp and homologs of the Ubi-proteins involved in the $\mathrm{O}_{2}$-independent UQ pathway are not present. This is in agreement with the strictly aerobic niche of this bacterium. Then, via two approaches, i.e. the use of an inhibitor (3-amino-4-hydroxybenzoic acid) and a transposon mutant, which both strongly impair the synthesis of UQ, we demonstrated that UQ is essential for the growth of $F$. novicida in a respiratory medium and contributes to its pathogenicity in Galleria mellonella used as an alternative animal model.

\section{Importance}

Francisella tularensis is the causative bacterium of tularemia and is classified as a biothreat agent. Using multidisciplinary approaches, we investigated the ubiquinone (UQ) biosynthetic pathway that operates in F. novicida used as a surrogate. We showed that $\mathrm{UQ}_{8}$ is the major quinone identified in the membranes of Francisella novicida. We identified a new competitive inhibitor, which strongly decreased the biosynthesis of UQ. Our demonstration of the crucial role of UQ for the respiratory metabolism of $F$. novicida and for the involving in its pathogenicity in the Galleria mellonella model should stimulate the search for selective inhibitors of bacterial UQ biosynthesis.

\section{Keywords}

Francisella novicida, Francisella tularensis, ubiquinone biosynthesis, respiratory chain, metabolism, pathogenicity.

\section{Introduction}

Francisella tularensis is a Gram-negative, strictly aerobic, facultative intracellular pathogen responsible for tularemia. Infection can occur by inhalation, ingestion, transmission from arthropod vectors or exposure to infected animals (1). After its entry into macrophages, the bacteria are sequestered into phagosomes and prevent further endosomal maturation. Francisella cells then disrupt the phagosome and are released into the cytosol in which they rapidly proliferate (2). Eventually, the infected cells undergo apoptosis or pyroptosis, and the progeny bacteria are released to initiate new rounds of infection (2). Currently, there is no suitable vaccine against tularemia and due to its extreme infectivity and high virulence, $F$. tularensis species have been classified as a biothreat agent (3). The genus Francisella includes the three species: $F$. tularensis, $F$. novicida and F. philomiragia (4). Moreover, $F$. 
tularensis is further divided into the subspecies tularensis (Type A strains) and holarctica (Type B strains), which are the most virulent strains responsible for human disease, whereas $F$. philomiragia and $F$. novicida are avirulent in healthy humans (4). A F. novicida type strain U112 is commonly used as a surrogate for Francisella tularensis in virulence studies using animal models (5).

The development of genome-scale genetic methods allowed the identification of hundreds of genes participating to variable extents to Francisella virulence (6). However, the specific contribution of only a limited number of these genes was demonstrated at the molecular level. Although an important proportion of the identified genes are related to metabolic functions, the relationship between metabolism and the life cycle of Francisella is still poorly understood. However, global analysis of genes essential for the growth in culture of F. novicida U112 (7) and more recently of that of F. tularensis ssp tularensis Schu S4 (8) highlighted the involvement of several ubiquitous pathways found in proteobacteria. Among the most significant are the folate pathway, the heme synthesis pathway, the methylerythritol phosphate pathway involved in isoprenoid synthesis, the chorismate pathway and the ubiquinone (UQ) synthesis pathway, on which this work is focused.

Isoprenoid quinones are conserved in most respiratory and photosynthetic organisms and function primarily as electron and proton carriers in the electron-transfer chains. Quinones are composed of a polar redox-active head group linked to a lipid side chain, which varies in both length and degree of saturation (9). Proteobacteria contain two main types of quinone, i.e. benzoquinones and naphthoquinones, represented by UQ (or coenzyme Q) and menaquinone (MK)/ demethylmenaquinone (DMK), respectively (9). UQ is the major electron carrier used for the reduction of dioxygen by various cytochrome oxidases, whereas MK and DMK function predominantly in anaerobic respiratory chains (9). However, as demonstrated recently in Pseudomonas aeruginosa, UQ can also be produced and used as a main respiratory quinone under anaerobic conditions (10). Besides its role in bioenergetics, UQ was also reported to be involved in gene regulation, oxidative stress, virulence and resistance to antibiotics $(11,12)$. More recently, new functions for UQ in bacteria were discovered such as its requirement for Escherichia coli to grow on medium containing longchain fatty acids as a carbon source (13). UQ biosynthesis in aerobic conditions has been widely studied in E. coli (14). The classical UQ biosynthetic pathway requires twelve proteins (UbiA to UbiK and UbiX). UbiC catalyzes the first committed step in the biosynthesis of UQ, the conversion of chorismate to the 4-hydroxybenzoate (4HB) precursor. Then, UbiA, UbiD 
100 to UbiI and UbiX catalyze the prenylation, decarboxylation, hydroxylations and methylations

101

102

103

104

105

106

107

108

109

110

111

112

113

114

115

116

117

118

119

120

121

122

123

124

125

126

127

128

129

130

131

132 of the phenyl ring of the 4HB to synthesize UQ. In addition, UbiB and UbiK are accessory proteins while UbiJ is involved in the assembly and/or the stability of the aerobic Ubicomplex, which was recently characterized in E. coli (15). This latter is able to synthesize UQ also under anoxic conditions and we identified three proteins, UbiU, UbiV and UbiT, which are required for UQ biosynthesis only under anoxic conditions (16).

Here, we show that $\mathrm{UQ}_{8}$ is the major quinone of $F$. novicida $\mathrm{U} 112$. We identified candidate Ubi-proteins in $F$. novicida U112 and validated their functions by heterologous complementation in E. coli mutant strains. Our results show that UQ biosynthesis in Francisella spp is mostly similar to that of E. coli, with the notable absence of UbiX and UbiD for the decarboxylation step. Genetic and chemical inactivation of UQ biosynthesis thanks to a transposon mutant and to a new inhibitor (3-amino-4-hydroxybenzoic acid), respectively, demonstrated that $\mathrm{UQ}_{8}$ is crucial for the growth of $F$. novicida in respiratory media and that UQ deficiency impairs the pathogenicity of $F$. novicida against Galleria mellonella. Altogether, our results shed light on the role of UQ in the life cycle of Francisella and show that UQ contributes to its pathogenicity.

\section{Results}

\section{$\mathrm{UQ}_{8}$ is the major quinone of $\boldsymbol{F}$. novicida.}

The quinone content of $F$. novicida grown under ambient air at $37^{\circ} \mathrm{C}$ in Chamberlain media supplemented with either glucose (fermentative medium) or succinate (respiratory medium) as the only carbon source was determined and compared with that of E. coli MG1655 grown in the same fermentative medium. In the electrochromatograms of lipid extracts from $F$. novicida, a single peak was observed around $8.3 \mathrm{~min}$, the same retention time as $\mathrm{UQ}_{8}$ in $E$. coli extracts (Fig. 1A). Note that in these analyses, $\mathrm{UQ}_{10}$ was used as an internal standard, which was added to the samples. MS analysis of the major peak in F. novicida extracts showed a predominant ammonium adduct $\left(\mathrm{M}^{+} \mathrm{NH}_{4}{ }^{+}\right)$with an $\mathrm{m} / \mathrm{z}$ ratio of 744.5 , together with minor adducts, such as $\mathrm{Na}^{+}$(749.7) and $\mathrm{H}^{+}$(727.8) (Fig. 1B). These masses identify $\mathrm{UQ}_{8}$ (monoisotopic mass, 726.5) as the major quinone produced by $F$. novicida. Interestingly, the carbon source in the culture media did not greatly affect the $\mathrm{UQ}_{8}$ content (Fig. 1A). The F. novicida extracts did not contain any naphthoquinones, unlike E. coli, which showed predominantly demethylmenaquinone $\left(\mathrm{DMK}_{8}\right)$ eluting around $12 \mathrm{~min}$. The absence of detectable levels of naphthoquinones in F. novicida lipid extracts (Fig. 1A) is in agreement 
with the absence of menaquinone biosynthesis (Men or futalosine) encoding genes in its genome. Together, our results establish that $E$. coli and $F$. novicida share $\mathrm{UQ}_{8}$ as a main quinone in aerobic conditions.

\section{Identification of Ubi-proteins in the Francisella spp.}

To identify candidate Ubi-proteins in F. novicida, UbiX, and UbiA to UbiK from $E$. coli MG1655 were screened for homologs in the protein sequence data set, available at MicroScope (www.genoscope.cns.fr/agc/microscope), using the BLASTP software. As listed in Table S1, this analysis identified eight homologous proteins in $F$. novicida, i.e. UbiA to UbiC, UbiE, UbiG to UbiI and UbiK, called hereafter $\mathrm{UbiA}_{F n}$ to $\mathrm{UbiC}_{F n}, \mathrm{UbiE}_{F n}, \mathrm{UbiG}_{F n}$ to $\mathrm{UbiI}_{F n}$ and $\mathrm{UbiK}_{F n}$, respectively. Genes $u b i A_{F n}$ and $u b i C_{F n}$ in one hand and genes $u b i I_{F n}$ and $u b i H_{F n}$ in another hand present an organization similar to the ubiC-ubiA and the ubiH-ubiI operons from E. coli, respectively (12). As reported previously for Pseudomonas aeruginosa (17) and Xanthomonas campestris (18), F. novicida possesses a Coq7 hydroxylase, which is a functional homolog of the UbiF protein found in E. coli and other species (19). The detection of an homolog for E. coli UbiJ required less restrictive Blast parameters. We noticed that the gene coding for the putative UbiJ candidate FTN_0460, hereafter called $u b i J_{F n}$, lies between $u b i E_{F n}$ and $u b i B_{F n}$, an organization similar to the $u b i E-u b i J-u b i B$ operon from E. coli (20). $\mathrm{UbiJ}_{F n}$ has $21 \%$ amino-acid identity with UbiJ from $E$. coli and both proteins contain a sterol carrier protein 2 domain in their N-terminal regions (http://pfam.xfam.org/) (21). The same Ubi-proteins were identified in the highly virulent strain F. tularensis ssp tularensis Schu S4 (Table S1).

Homologs of UbiD and UbiX were not yet identified and the counterparts of these two proteins in Francisella spp remain to be determined. The work is in progress in our laboratory. Under anaerobic conditions, E. coli still synthesizes UQ, and we recently identified three genes, which we called $u b i T, u b i U$, and $u b i V$, as essential for this process (16). Homologs of $u b i T$, ubiU, and $u b i V$, which participate to the $\mathrm{O}_{2}$-independent UQ biosynthetic pathway, were not identified in the screened Francisella genomes (Table S1), in agreement with the strictly aerobic metabolism of Francisella spp. In overall, these data show that the $\mathrm{O}_{2}$-dependent UQ biosynthetic pathways in $F$. novicida, in F. tularensis and in E. coli are related, the major difference being the absence of UbiX-UbiD for the decarboxylation step (Fig. S1). 


\section{Functional characterization of $U b i_{F n}$-proteins in $E$. coli.}

To test whether the candidate Ubi-proteins identified in F. novicida were indeed involved in UQ biosynthesis, we assessed their capacity to functionally complement $E$. coli strains in which the UQ protein-encoding genes were inactivated ( $\triangle u b i A c, \Delta u b i B c, \Delta u b i C c$, $\Delta u b i E c, \Delta u b i F c, \Delta u b i G c, \Delta u b i H c, \Delta u b i I c, \Delta u b i J$ and $\Delta u b i K c$, see Table S2). We assessed the quinone content and the capacity to grow on solid minimal medium containing fermentable (glucose) or respiratory (succinate) carbon sources. E. coli $\Delta u b i A c, \Delta u b i B c, \Delta u b i G c, \Delta u b i H c$ and $\Delta u b i J$ transformed with empty vector are unable to synthesize $\mathrm{UQ}_{8}$ (Fig. 2A) and are thus unable to grow on a respiratory medium (Fig. 2C). In contrast, their growth on a fermentative medium is not affected (Fig. 2C). Except for the $\triangle u b i A c$ mutant strain, in which the prenylation reaction of the $4 \mathrm{HB}$ is impaired, most mutants accumulate an early intermediate corresponding to octaprenylphenol (OPP) (Fig. 2A and S1). E. coli $\triangle u b i E c$ and $\Delta u b i F c$ cells accumulate C2-demethyl-C6-demethoxy-UQ $\mathrm{UD}_{8}\left(\mathrm{DDM}_{8}\right)$ and C6-demethoxy-UQ ${ }_{8}\left(\mathrm{DMQ}_{8}\right)$, which are the substrates of $\mathrm{UbiE}$ and $\mathrm{UbiF}$, respectively (Fig. 2A and S1). We found that $\mathrm{UbiA}_{F n}, \mathrm{UbiB}_{F n}, \mathrm{UbiE}_{F n}, \mathrm{Coq}_{F n}$ and $\mathrm{UbiG}_{F n}$ restored growth of E. coli $\Delta u b i A c, \Delta u b i B c$, $\triangle u b i E c, \triangle u b i F c$ and $\triangle u b i G c$ cells on respiratory medium (Fig. 2C) and allowed for $\mathrm{UQ}_{8}$ biosynthesis in LB medium to 96, 26, 7, 49 and 38\% of the level of $\mathrm{UQ}_{8}$ present in the WT cells, respectively (Fig. 2A and 3A). Concomitantly, OPP content decreased and Coq7 $F n$ abolished the accumulation of $\mathrm{DMQ}_{8}$ in $\triangle u b i F c$ cells (Fig. 2A). As we previously reported, $E$. coli $\triangle u b i I c$ and $\Delta u b i K c$ cells displayed a strong decrease in $\mathrm{UQ}_{8}(22,23)$, but the residual $\mathrm{UQ}_{8}$ content was sufficient to support growth on succinate (Fig. 2A and 2C). Similar results were obtained with $\triangle u b i C c$ cells grown in minimal M9 medium (Fig. 2B and 2C), which had to be used instead of $\mathrm{LB}$ since the later contains $4 \mathrm{HB}$ that restores normal $\mathrm{UQ}_{8}$ content in $\Delta u b i C c$ (data not shown). In all three strains, the expression of the corresponding Ubiproteins, $\mathrm{UbiC}_{F n}, \mathrm{UbiI}_{F n}$ and $\mathrm{UbiK}_{F n}$, increased significantly the $\mathrm{UQ}_{8}$ content (Fig. $3 \mathrm{~A}$ and 3B). Since the increase obtained in $\Delta u b i I c$ cells was moderate (from 25 to 40\%), we further confirmed the ability of $\operatorname{UbiI}_{F n}$ to catalyze C5-hydroxylation by using an E. coli $\Delta u b i I c \Delta u b i F$ strain. This deletion mutant lacks C5- and C6-hydroxylation activities and consequently accumulates 3-octaprenyl-4-hydroxyphenol $\left(4 \mathrm{HP}_{8}\right)(22)$. We found that $\mathrm{UbiI}_{F n}$ was able to restore $\mathrm{DMQ}_{8}$ biosynthesis in E. coli $\Delta u b i I c \Delta u b i F$ cells (Fig. S2), i.e. to catalyze C5hydroxylation, concomitantly to a strong decrease of $4 \mathrm{HP}_{8}$. Taken together, all these results confirm unambiguously that $\mathrm{UbiA}_{F n}, \mathrm{UbiB}_{F n}, \mathrm{UbiC}_{F n}, \mathrm{Coq}_{F n}, \mathrm{UbiE}_{F n}, \mathrm{UbiG}_{F n}, \mathrm{UbiI}_{F n}$ and 
$\mathrm{UbiK}_{F n}$ are the functional counterpart of the E. coli Ubi-proteins and we propose that they compose the biosynthetic pathway of $\mathrm{UQ}_{8}$ in $F$. novicida. Only two proteins, $\mathrm{UbiJ}_{F n}$ and $\mathrm{UbiH}_{F n}$ did not complement the E. coli $\Delta u b i J$ and $\Delta u b i H c$ (Fig. 2A, 2C and 3A). The low percentage of identity between UbiJ and UbiH from E. coli and their homologs in F. novicida (21 and 27\%, respectively) could explain these results (Table S1).

\section{$\mathrm{UQ}_{8}$ biosynthesis is essential for the growth of $F$. novicida in respiratory medium.}

To evaluate the physiological importance of UQ for $F$. novicida, we screened $u b i$ genes in the F. novicida transposon (Tn) mutant library available at the Manoil Laboratory (7). Only the Tn mutant of $u b i C_{F n}$ (called hereafter Tn- $u b i C_{F n}$ ) was available in the library, and we compared this mutant strain to its isogenic control strain U112 (Table S2). Recall that UbiC catalyzes the first committed step in the biosynthesis of UQ, i.e. the conversion of chorismate to $4 \mathrm{HB}$ (Fig. S1). First, we showed that the growth of Tn-ubiC $C_{F n}$ cells under ambient air in respiratory Chamberlain medium was severely impaired compared to the WT (Fig. 4A). In contrast, the growth of F. novicida in fermentative medium was less affected (Fig. 4B). In parallel, $\mathrm{UQ}_{8}$ content was strongly lowered in Tn- $u b i C_{F n}$ cells from 166 to 7 $\mathrm{pmol} / \mathrm{mg}$ cells in fermentative medium and from 134 to $11 \mathrm{pmol} / \mathrm{mg}$ cells in respiratory medium (Fig. 4C). As expected, addition of 4HB to the culture rescued the growth of Tn$u b i C_{F n}$ in respiratory medium and increased the $\mathrm{UQ}_{8}$ content to WT levels (Fig. 4C). Taken together, these results show the overall requirement of $\mathrm{UQ}_{8}$ for the growth of $F$. novicida especially in respiratory medium.

3A4HB inhibits $\mathrm{UQ}_{8}$ biosynthesis and impairs the growth of $F$. novicida in respiratory medium.

Besides genetic inactivation of the UQ pathway, we were interested in the possibility to decrease UQ levels by chemical inhibition. Since we had found UQ to be particularly important for growth of $F$. novicida in respiratory medium (Fig. 4A), we screened for compounds that could inhibit growth in such medium. We tested several compounds: 3amino-4-hydroxybenzoic acid (3A4HB), 4-amino-benzoic acid (pABA), 4-amino-2-methoxybenzoic acid (pA2MBA) and 4-amino-3-methoxy-benzoic acid (pA3MBA). All these molecules are analogs of $4 \mathrm{HB}$, the native precursor of UQ (Fig. S3A). We observed that pA2MBA, while pA3MBA inhibited growth both in fermentative and respiratory media (Fig. 
S3B-C). Interestingly, 3A4HB strongly impaired bacterial growth in respiratory medium, while inhibition was milder in fermentative medium (Fig. S3B-C). Based on these results, we followed up on this compound.

We then examined how and to what extent $3 \mathrm{~A} 4 \mathrm{HB}$ could affect $\mathrm{UQ}_{8}$ biosynthesis in $F$. novicida. Bacteria were cultured under ambient air in fermentative Chamberlain medium supplemented with $3 \mathrm{~A} 4 \mathrm{HB}$ (from $10 \mu \mathrm{M}$ to $1 \mathrm{mM}$, final concentration). Endogenous $\mathrm{UQ}_{8}$ content was measured in the bacterial cells and compared to a control condition in which only DMSO was added. Figure $5 \mathrm{~A}$ shows that the $\mathrm{UQ}_{8}$ content decreased with increasing concentrations of $3 \mathrm{~A} 4 \mathrm{HB}$ in the medium, with $0.5 \mathrm{mM}$ yielding to a $\sim 90 \%$ decrease of the $\mathrm{UQ}_{8}$ content. Concomitantly, we confirmed that growth of $F$. novicida in presence of $1 \mathrm{mM}$ 3A4HB was strongly impaired in respiratory medium (Fig. 5B), but less so in a fermentative medium (Fig. 5C). Control experiments showed that addition of $4 \mathrm{HB}$ to the growth medium counteracted the negative effect of $3 \mathrm{~A} 4 \mathrm{HB}$, both in terms of $\mathrm{UQ}_{8}$ biosynthesis and bacterial growth (Fig. 5A to 5C).

Treatment with $3 \mathrm{~A} 4 \mathrm{HB}$ caused the accumulation of a redox compound that eluted at 6.5 min (compound $\mathrm{X}$ on Fig. 5A). MS analysis of this peak showed a predominant proton adduct $\left(\mathrm{M}^{+} \mathrm{H}^{+}\right)$with an $\mathrm{m} / \mathrm{z}$ ratio of 682.6 , together with a minor sodium adduct $\left(\mathrm{M}^{+} \mathrm{Na}^{+}\right)$ with an $\mathrm{m} / \mathrm{z}$ ratio of 704.6 (Fig. 5D). Both species are compatible with a monoisotopic mass of 681.7 g.mol ${ }^{-1}$, which could correspond to that of 2-octaprenyl-3-methyl-6-amino-1,4benzoquinone (Fig. 5E). According to the sequence of reactions proposed in Figure S1, the formation of compound $\mathrm{X}$ would result from prenylation of $3 \mathrm{~A} 4 \mathrm{HB}$, decarboxylation and hydroxylation at $\mathrm{C} 1$ and then methylation at C3. Thus, 3-octaprenyl-2-methyl-5-amino-1,4benzoquinone seems to be the "dead-end" product of the UQ8 pathway in $F$. novicida cells treated with $3 \mathrm{~A} 4 \mathrm{HB}$. Collectively, these results demonstrate unequivocally that $3 \mathrm{~A} 4 \mathrm{HB}$ acts as a competitive inhibitor of $\mathrm{UQ}_{8}$ biosynthesis and affects particularly the respiratory metabolism of $F$. novicida.

\section{$\mathrm{UQ}_{8}$ is involved in the pathogenesis of $F$. novicida in the later steps of the infection.}

We evaluated the importance of UQ in the pathogenicity of F. novicida by studying the $\mathrm{Tn}-u b i C_{F n}$ mutant. To assess the overall virulence of the Tn- $u b i C_{F n}$ strain in a whole organism, we used the wax moth (G. mellonella) infection model, which was previously used in studies of human pathogenic and closely related opportunistic and non-pathogenic Francisella spp, such as F. novicida (24-27). We monitored the survival of larvae infected 
with the Tn- $u b i C_{F n}$ strain or with the isogenic strain U112 as control. When the larvae are turning grey/black and no movement of the larval legs can be observed, they are considered dead (Fig. 6A). The Tn-ubiC $C_{F n}$ strain was found to be statistically much less virulent than the wild type, but was nevertheless still capable to kill Galleria larvae (Fig. 6B). This result suggests that $\mathrm{UQ}_{8}$ is involved in the virulence potential of $F$. novicida in G. mellonella. To better understand the role of $\mathrm{UQ}_{8}$ in the different stages of infection in G. mellonella, the pathogenicity of the isogenic control strain pre-treated with $1 \mathrm{mM} 3 \mathrm{~A} 4 \mathrm{HB}$ was studied in order to mimic a $\mathrm{UQ}_{8}$ acute deficiency. Recall that this treatment causes a $\sim 90 \%$ decrease of the $\mathrm{UQ}_{8}$ content (Fig. 5A) but the inhibition should be alleviated over the infection cycle in the larvae where $3 \mathrm{~A} 4 \mathrm{HB}$ is not present. The pretreatment with $3 \mathrm{~A} 4 \mathrm{HB}$ has no effect on the capacity of F. novicida to kill Galleria larvae (Fig. 6C), suggesting that $\mathrm{UQ}_{8}$ does not contribute to the virulence of Francisella novicida in the early steps of the infection but more likely in later ones. This result contrasts with that obtained with the Tn- $u b i C_{F n}$ strain, which represents a chronic deficiency of $\mathrm{UQ}_{8}$.

\section{Discussion}

The chemical analysis performed in this paper established that $\mathrm{UQ}_{8}$ is the major isoprenoid quinone synthesized by $F$. novicida. In two representative Francisella genomes, we identified homologs for nine of the twelve genes, which are currently known to contribute to UQ biosynthesis in E. coli under aerobic conditions. We confirmed the function of seven of the nine homologs by heterologous complementation of E. coli $\Delta u b i$ mutants. From these results, we show again that $E$. coli is a good model to study the function of most exogenous $u b i$-genes (19). We could not confirm the function of $\mathrm{UbiH}_{F n}$ and $\mathrm{UbiJ}_{F n}$, but the fact that $u b i H_{F n}$ and $u b i J_{F n}$ show the same genetic organization as in E. coli (a $u b i I-u b i H$ operon and a $u b i E-u b i J-u b i B$ operon) strongly supports the implication of these genes in the UQ biosynthetic pathway. Interestingly, both proteins are part of the Ubi-complex in E. coli (15).

We hypothesize that the low identity of $\mathrm{UbiH}_{F n}$ and $\mathrm{UbiJ}_{F n}$ with their $E$. coli homologs ( $25 \%$ ) might impair their assembly within the E. coli Ubi-complex and thus compromise our in vivo complementation assays. Another possibility relates to the proposed implication in UQ biosynthesis of a non-coding RNA partially overlapping the ORF of UbiJ from E. coli (28). We note that the expression of UbiJ from $X$. campestris was also unable to complement an $E$. coli $\Delta$ ubiJ strain (18). Francisella spp shares with $P$. aeruginosa and X. campestris a yeast Coq7 protein homolog, which catalyzes the C6-hydroxylation as UbiF from E. coli (17, 18, 
29). As we demonstrated previously, the Coq7 proteins are found in all three subclasses, alpha-, beta- and gamma-proteobacteria. In contrast, homologs of UbiF proteins are limited to the gamma-proteobacteria (19). Our analysis disclosed also the presence in Francisella spp of UbiI and UbiH homologous proteins, which catalyze C5- and C1-hydroxylation in E. coli, respectively $(22,30)$. Consequently, we propose that both $E$. coli and Francisella spp share a UQ biosynthetic pathway involving three hydroxylases, i.e. UbiI, UbiH and UbiF in E. coli or UbiI, UbiH and Coq7 in Francisella spp. Several studies highlighted that the enzymes involved in multiple steps of the UQ biosynthetic pathway vary between bacterial species (14), like for the hydroxylation steps (19), or for the production of 4HB from chorismate by UbiC or XanB2 proteins (31). The decarboxylation step involves UbiD and UbiX in E. coli (32), but we could not identify homologs in Francisella genomes. A candidate gene ubiZ was proposed based on its co-localization with $u b i E$ and $u b i B$ in the genomes of Acinetobacter spp and Psychrobacter sp. PRwf-1, which are also devoid of homologs of UbiD and UbiX (33). However, ubiZ was not confirmed functionally and this gene is not conserved in Francisella genomes. This suggests the existence of another decarboxylation system operating in UQ biosynthesis in Francisella spp and potentially in other bacteria lacking $u b i X$ and $u b i D$ (34).

To assess the essentiality of the UQ biosynthetic pathway in the respiratory metabolism of $F$. novicida, two different approaches were carried out. First, we showed that a transposon mutation of $u b i C_{F n}$ gene, which decreases 4HB synthesis, impaired the growth of F. novicida mainly in respiratory medium. Interestingly, among all the $u b i$-gene identified in Francisella genomes, only ubiC was mutated in large-scale studies $(7,8)$. This supports that the other ubi genes are essential for the viability of Francisella spp and strengthens the idea that UQ is key for the development of these bacteria. We noted that the mutation of the ubiC gene affects more severely $F$. novicida than $E$. coli for growth in respiratory media, despite both mutants producing comparable amounts of UQ. ( 7-8\% compared to the WT) (Fig. 3B and 5A). As E. coli synthesizes naphthoquinones but $F$. novicida does not, we propose that the milder phenotype of the $E$. coli ubiC mutant results from naphthoquinones participating to aerobic respiration, as previously suggested (35). Second, we tested the effect of structural analogs of $4 \mathrm{HB}$ and we showed that $3 \mathrm{~A} 4 \mathrm{HB}$ impaired the growth of $F$. novicida mainly in respiratory medium in agreement with a strong decrease of $\mathrm{UQ}_{8}$ biosynthesis. We demonstrated that $3 \mathrm{~A} 4 \mathrm{HB}$ competes with endogenous $4 \mathrm{HB}$ and progresses through several steps of the UQ biosynthetic pathway to form the redox compound $\mathrm{X}$ that we propose to be 3octaprenyl-2-methyl-5-amino-1,4-benzoquinone. As the $\mathrm{Tn}-u b i C_{F n}$ strain and the control 
strain U112 treated with $1 \mathrm{mM} 3 \mathrm{~A} 4 \mathrm{HB}$ yielded both to a $~ 90 \%$ decrease of the $\mathrm{UQ}_{8}$ content and presented a strong impairment of the growth in a respiratory medium (Fig. 4 and 5), we propose that compound $\mathrm{X}$ would not be used as a quinone in the respiratory chain of $F$. novicida.

We noted that homologs of $u b i T$, ubiU, and $u b i V$, which belongs to the $\mathrm{O}_{2-}$ independent UQ biosynthetic pathway characterized in E. coli and P. aeruginosa $(10,16)$, were not identified in the screened genomes of Francisella spp. This result is in agreement with the strictly aerobic metabolism of these bacteria. Indeed, tricarboxylic acid (TCA) cycle and the UQ-dependent electron-transfer chain, leading to efficient oxidative phosphorylation, take place in Francisella spp (36). A possible link between stress defense and the TCA cycle was previously suggested in Francisella pathogenesis (37). Unfortunately, the contribution of UQ and the electron-transfer chain to virulence has not been well documented to date in Francisella spp. Using G. mellonella as infection model at the scale of an entire organism, we demonstrated through the study of the Tn- $u b i C_{F n}$ mutant and the isogenic control strain pretreated with $3 \mathrm{~A} 4 \mathrm{HB}$, that $\mathrm{UQ}_{8}$ contributes to the virulence of $F$. novicida and more likely in the later steps of the infection, during which the bacteria undergo extensive replication (38). Such a notion support the view that, as other facultative intracellular bacteria, Francisella spp are able to use several substrates in order to grow in various environments, such as macrophages. Glycerol via the gluconeogenesis and amino acids were identified as main sources of carbon during intracellular replication of Francisella spp in the host cells $(36,39)$. However, glycerol requires UQ to be efficiently metabolized via the ubiquitous enzyme GlpD (40) and amino acids degradation is closely linked to the TCA cycle, which produces reducing equivalents in Francisella spp (36). Besides its requirement for bioenergetics, UQ might also contribute to the antioxidant capacity of Francisella since it was shown to be a potent lipid soluble antioxidant in E. coli (41). During its intracellular life, Francisella is exposed to oxidative stress. Indeed, as a defense mechanism for the clearance of phagocytosed microorganisms, both macrophages and neutrophils produce reactive oxygen species, which in turn trigger bacterial killing by causing damage to macromolecules $(42,43)$. We propose that the reduced content in UQ in the Tn- $u b i C_{F n}$ mutant could therefore affect $F$. novicida's oxidative defense. This hypothesis is in good agreement with recent data showing that reduced expression of $\mathrm{UbiC}_{F n}$ decreases the resistance of $F$. novicida to oxidative stress (44). In a similar way, we showed previously that UbiE, UbiJ and UbiB proteins were needed in Salmonella enterica serovar Typhimurium intracellular proliferation in macrophages (21). 
Collectively, all these data assign a role for Ubi-proteins in bacterial intracellular proliferation and, more generally, highlight the importance of UQ production for bacterial virulence.

\section{Materials and methods}

\section{Bacterial strains and growth conditions}

All bacterial strains used in this study are listed in Table S2. F. novicida U112 was obtained from the Centre National de Référence des Francisella, CHU Grenoble-Alpes, France. The transposon mutant $\mathrm{Tn}-u b i C_{F n}$ in the $F$. novicida $\mathrm{U} 112$ strain was obtained from the Manoil Laboratory, Department of Genome Science, University of Washington (7). Both strains were grown on Polyvitex enriched chocolate agar plates (PVX-CHA, bioMérieux, Marcy L'Etoile, France) incubated at $37^{\circ} \mathrm{C}$ for $48-72$ h. Liquid cultures were carried out at $37^{\circ} \mathrm{C}$ with rotary shaking at $200 \mathrm{rpm}$ in Chamberlain medium (45) supplemented with either glucose or succinate $(0.4 \%$ (wt/vol) final concentration) as the only carbon source. For growth studies, overnight cultures were used to inoculate a 96-well plate to obtain a starting optical density at $600 \mathrm{~nm}$ (OD600) of around 0.1 and further incubated under shaking at $37^{\circ} \mathrm{C}$. Changes in $\mathrm{OD}_{600}$ were monitored every $10 \mathrm{~min}$ for $40 \mathrm{~h}$ using the Infinite 200 PRO microplate reader (Tecan, Lyon, France). When required, the medium was supplemented with 4HB in DMSO at 50-100 $\mu \mathrm{M}$ final concentration, pABA, pA2MBA and pA3MBA in DMSO at $1 \mathrm{mM}$ final concentration or $3 \mathrm{~A} 4 \mathrm{HB}$ at $10 \mu \mathrm{M}-1 \mathrm{mM}$, final concentration. For $\mathrm{CFU}$ counting, bacteria were suspended in PBS and cell suspensions were serially diluted in PBS. For each sample, $100 \mu \mathrm{L}$ of at least four different dilutions were plated on PVX-CHA plates and incubated for $72 \mathrm{~h}$ at $37^{\circ} \mathrm{C}$, and CFU were counted using a Scan 100 Interscience.

The E. coli $\triangle u b i A$ and $\Delta u b i J$ mutants were constructed as described previously (46). Briefly, the ubiA::cat and ubiJ::cat mutation was generated in a one-step inactivation of the ubiA and ubiJ genes. A DNA fragment containing the cat gene flanked with the 5' and 3' regions of the ubiA and ubiJ genes was PCR amplified using pKD3 as a template and oligonucleotides 5'-wannerubiA/3'-wannerubiA and 5'-wannerubiJ/3'-wannerubiJ, respectively (Table S3). The $\triangle u b i B$ mutant was generated as follows: the cat gene was inserted in $u b i B$ gene between the two sites NruI at 842 and $1004 \mathrm{pb}$. Then, ubiB::cat was PCR amplified using oligonucleotides 5'-xbaIubiB/3'-xbaIubiB (Table S3). Strain BW25113, carrying the pKD46 plasmid, was transformed by electroporation with the amplified fragments and $\mathrm{Cat}^{\mathrm{r}}$ colonies were selected. The replacement of chromosomal $u b i$ by the $c a t$ 
396

397

398

399

400

401

402

403

404

405

406

407

408

409

410

411

412

413

414

415

416

417

418

419

420

421

422

423

424

425

426

427

428

gene was verified by PCR amplification in the Cat ${ }^{\mathrm{r}}$ clones. E. coli $\mathrm{K}-12$ strains JW5713 and JW2226 from the Keio Collection (47) were used as donors in transduction experiments to construct the $\triangle u b i C:: k a n$ and $\Delta u b i G:: k a n$ mutants of E. coli MG1655 strains. The $\Delta u b i A$, $\triangle u b i B, \Delta u b i C, \triangle u b i E, \triangle u b i G$ and $\triangle u b i K$ strains were cured with pCP20 to yield $\triangle u b i A c$, $\triangle u b i B c, \triangle u b i C c, \Delta u b i E c, \Delta u b i G c$ and $\Delta u b i K c$ strains, respectively (Table S2). E. coli strains (K12, MG1655 or Top10) were grown on lysogeny broth (LB)-rich medium or in M9 minimal medium (supplemented with glucose or succinate, $0.4 \%(\mathrm{wt} / \mathrm{vol})$ final concentration) at $37^{\circ} \mathrm{C}$. Ampicillin $(100 \mu \mathrm{g} / \mathrm{ml})$, kanamycin $(50 \mu \mathrm{g} / \mathrm{ml})$, chloramphenicol $(35 \mu \mathrm{g} / \mathrm{ml})$ and IPTG $(100 \mu \mathrm{M})$ were added when needed.

\section{Cloning, plasmid construction, and complementation assays.}

The plasmids and the primers used in this study are listed in Tables S2 and S3 (supplemental material), respectively. All the plasmids produced in this work were verified by DNA sequencing (GATC Biotech, Konstanz, Germany). The FTN_0385 (ubiA $F n$ ), FTN_0459 $\left(u b i B_{F n}\right), F T N \_0386\left(u b i C_{F n}\right), F T N \_0461\left(u b i E_{F n}\right), F T N \_1146\left(\operatorname{coq} 7_{F n}\right), F T N \_0321\left(u b i G_{F n}\right)$, $F T N \_1237\left(u b i H_{F n}\right), F T N \_1236\left(u b i I_{F n}\right), F T N \_0460\left(u b i J_{F n}\right)$ and $F T N_{-} 1666\left(u b i K_{F n}\right)$ inserts were obtained by PCR amplification using the $F$. novicida $\mathrm{U} 112$ genome as template and the oligonucleotides described in Table S3. Inserts were EcoRI-BamHI or EcoRI-HindIII digested and inserted into EcoRI-BamHI- or EcoRI-HindIII-digested pTrc99a plasmids, respectively,

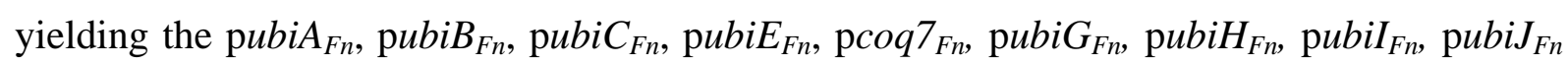
and $\mathrm{p} u b i K_{F n}$ plasmids (Table S3). The plasmids were transformed into E. coli MG1655 strains with mutation of the ubiA, ubiB, ubiC, ubiE, ubiF, ubiG, ubiH, ubiI, ubiJ and ubiK genes (single and double mutations, see Table S2), and complementation of the $\mathrm{UQ}_{8}$ biosynthetic defect was assessed by both measuring the quinone content and plating serial dilutions onto solid M9 minimal medium supplemented with glucose or succinate $(0.4 \%$ (wt/vol) final concentration) as the only carbon sources and overnight growth at $37^{\circ} \mathrm{C}$. Expression of the Ubi-proteins was induced by addition of IPTG to a final concentration of $100 \mu \mathrm{M}$.

\section{Lipid extractions and quinone analysis}

Cultures (5 $\mathrm{ml}$ under ambient air) were cooled down on ice $30 \mathrm{~min}$ before centrifugation at $3200 \mathrm{Xg}$ at $4{ }^{\circ} \mathrm{C}$ for $10 \mathrm{~min}$. Cell pellets were washed in $1 \mathrm{ml}$ ice-cold PBS and transferred to preweighted $1.5 \mathrm{~mL}$ Eppendorf tubes. After centrifugation at 12,000 g at 4 ${ }^{\circ} \mathrm{C}$ for $1 \mathrm{~min}$, the supernatant was discarded, the cell-wet weight was determined $(\sim 5-30 \mathrm{mg})$ 
and pellets were stored at $-20^{\circ} \mathrm{C}$. Quinone extraction from cell pellets was performed as previously described (22). Lipid extracts corresponding to $1 \mathrm{mg}$ of cell-wet weight were analyzed by HPLC electrochemical detection-MS (ECDMS) with a BetaBasic-18 column at a flow rate of $1 \mathrm{~mL} / \mathrm{min}$ with mobile phases composed of 50\% methanol, $40 \%$ ethanol, and a mix of $90 \%$ isopropanol, $10 \%$ ammonium acetate $(1 \mathrm{M})$, and $0.1 \%$ TFA). When necessary, MS detection was performed on an MSQ spectrometer (Thermo Scientific) with electrospray ionization in positive mode (probe temperature, $400^{\circ} \mathrm{C}$; cone voltage, $80 \mathrm{~V}$ ). Single-ion monitoring detected the following compounds: $\mathrm{UQ}_{8}\left(\mathrm{M}^{+} \mathrm{NH} 4^{+}\right), \mathrm{m} / \mathrm{z}$ 744-745, 6-10 min, scan time of $0.2 \mathrm{~s}$; $\mathrm{UQ}_{10}\left(\mathrm{M}^{+} \mathrm{NH} 4^{+}\right), \mathrm{m} / \mathrm{z} 880-881,10-17 \mathrm{~min}$, scan time of $0.2 \mathrm{~s} ; \mathrm{DMQ}_{8}\left(\mathrm{M}^{+}\right.$ $\left.\mathrm{NH}_{4}^{+}\right), \mathrm{m} / \mathrm{z}$ 714-715-10 min, scan time of $0.4 \mathrm{~s}$; $\mathrm{DDMQ}_{8}\left(\mathrm{M}^{+} \mathrm{NH} 4^{+}\right), \mathrm{m} / \mathrm{z}$ 700-701, 5-8 min, scan time of 0.4 s; OPP $\left(\mathrm{M}^{+} \mathrm{NH} 4^{+}\right), 656.0-657,5-9 \mathrm{~min}$, scan time of $0.4 \mathrm{~s}$; compound $\mathrm{X}\left(\mathrm{M}^{+}\right.$ $\left.\mathrm{H}^{+}\right), \mathrm{m} / \mathrm{z} 682-683,5-10 \mathrm{~min}$, scan time of $0.4 \mathrm{~s}$. MS spectra were recorded between $\mathrm{m} / \mathrm{z} 600$ and 900 with a scan time of $0.3 \mathrm{~s}$. ECD and MS peak areas were corrected for sample loss during extraction on the basis of the recovery of the $\mathrm{UQ}_{10}$ internal standard and then were normalized to cell wet weight. The peaks of $\mathrm{UQ}_{8}$ obtained with electrochemical detection or MS detection were quantified with a standard curve of $\mathrm{UQ}_{10}$ as previously described (22).

\section{Infections of $G$. mellonella larvae}

Larvae of the wax moth G. mellonella were purchased from Lombri'carraz SARL, Mery, France. Healthy and uniformly white larvae measuring around $3 \mathrm{~cm}$ were selected for infection. The bacteria were grown over night to an $\mathrm{OD}_{600}$ of around three. Culture medium was removed by centrifugation and bacteria were diluted in PBS to $10^{8} \mathrm{CFU} / \mathrm{mL}$. Insulin cartridges were sterilized before filling with bacterial solutions. Larvae were injected with 10 $\mu \mathrm{L}$ of bacterial suspensions $\left(10^{6} \mathrm{CFU}\right.$ per larva as recommended (24)) using an insulin pen or with $10 \mu \mathrm{L}$ of PBS only. The precise number of bacteria transferred in injections was determined by spotting serial dilutions onto chocolate agar plates, and counting CFU after growth at $37^{\circ} \mathrm{C}$ for $48 \mathrm{~h}$. Infected larvae were placed in Petri dishes and maintained at $37^{\circ} \mathrm{C}$. Survival of larvae was monitored for 6 days by counting the number of dead larvae each day. A cohort of 20 larvae was used per condition, and the experiment was performed twice. As a control, an untreated cohort of larvae was also followed.

\section{Acknowledgments}


461 This work was supported by the Agence Nationale de la Recherche (ANR), project O2-taboo 462 ANR-19-CE44-0014, project Emergence (TIMC-UGA), the University Grenoble Alpes 463 (UGA) and the French Centre National de la Recherche Scientifique (CNRS). We thank Dr 464 Patricia Renesto for constructive discussions, technical assistance and Professor Laurent 465 Aussel for critically reading the paper.

466

467

468

469

470

471

472

473

474

475

476

477

478

479

480

481

482

483

484

485

486

487

488

491 489 interest in Francisella tularensis. Nat Rev Microbiol 2:967-978.

490 4. Sjostedt A. 2007. Tularemia: history, epidemiology, pathogen physiology, and clinical

492 5. Kingry LC, Petersen JM. 2014. Comparative review of Francisella tularensis and 493 Francisella novicida. Front Cell Infect Microbiol 4:35.

\section{Contributions}

F.B., F.P., and L.P. conceived the project and its design. K.K., M.H.C. and G.H. conducted experiments and performed data analysis. K.K., C.B. and Y.C. performed experiments on $G$. mellonella. L.L. contributed to new reagents (strains). All authors edited the manuscript. L.P. wrote the manuscript. L.P. supervised the project.

\section{Abbreviations}

The abbreviations used are: $\mathrm{MK}_{8}$, menaquinone 8; $\mathrm{DMK}_{8}$ dimethyl-menaquinone 8; OHB, 3octaprenyl-4-hydroxybenzoic acid; OPP, octaprenylphenol; DMQ, C6-demethoxyubiquinone 8; $\mathrm{DDMQ}_{8}, \mathrm{C} 1$-demethyl-C6-demethoxy-ubiquinone 8; $\mathrm{UQ}_{8}$, ubiquinone 8; 3A4HB, 3-amino-4-hydroxybenzoic acid; pABA, 4-amino-benzoic acid, pA2MBA, 4-amino2-methoxy-benzoic acid and pA3MBA, 4-amino-3-methoxy-benzoic acid; ECD, electrochemical detection; Tn, transposon; $\mathrm{OD}_{600}$, optical density at $600 \mathrm{~nm}$ and IPTG, isopropyl-1-thio- $\beta$-D-galactopyranoside.

\section{References}

1. Sjostedt A. 2011. Special topic on Francisella tularensis and tularemia. Front Microbiol 2:86.

2. Wallet P, Lagrange B, Henry T. 2016. Francisella Inflammasomes: Integrated responses to a cytosolic stealth bacterium. Curr Top Microbiol Immunol 397:229-256.

3. Oyston PC, Sjostedt A, Titball RW. 2004. Tularaemia: bioterrorism defence renews 91 manifestations. Ann N Y Acad Sci 1105:1-29. 
6. Jones BD, Faron M, Rasmussen JA, Fletcher JR. 2014. Uncovering the components of the Francisella tularensis virulence stealth strategy. Front Cell Infect Microbiol 4:32.

7. Gallagher LA, Ramage E, Jacobs MA, Kaul R, Brittnacher M, Manoil C. 2007. A comprehensive transposon mutant library of Francisella novicida, a bioweapon surrogate. Proc Natl Acad Sci USA 104:1009-1014.

8. Ireland PM, Bullifent HL, Senior NJ, Southern SJ, Yang ZR, Ireland RE, Nelson M, Atkins HS, Titball RW, Scott AE. 2019. Global analysis of genes essential for Francisella tularensis Schu S4 growth in vitro and for fitness during competitive infection of fischer 344 rats. J Bacteriol 201:e00630-18.

9. Nowicka B, Kruk J. 2010. Occurrence, biosynthesis and function of isoprenoid quinones. Biochim Biophys Acta 1797:1587-1605.

10. Vo CD, Michaud J, Elsen S, Faivre B, Bouveret E, Barras F, Fontecave M, Pierrel F, Lombard M, Pelosi L. 2020. The $\mathrm{O}_{2}$-independent pathway of ubiquinone biosynthesis is essential for denitrification in Pseudomonas aeruginosa. J Biol Chem 295:90219032.

11. Soballe B, Poole RK. 1999. Microbial ubiquinones: multiple roles in respiration, gene regulation and oxidative stress management. Microbiology (Reading) 145:1817-1830.

12. Aussel L, Pierrel F, Loiseau L, Lombard M, Fontecave M, Barras F. 2014. Biosynthesis and physiology of coenzyme $\mathrm{Q}$ in bacteria. Biochim Biophys Acta 1837:1004-1011.

13. Agrawal S, Jaswal K, Shiver AL, Balecha H, Patra T, Chaba R. 2017. A genome-wide screen in Escherichia coli reveals that ubiquinone is a key antioxidant for metabolism of long-chain fatty acids. J Biol Chem 292:20086-20099.

14. Abby SS, Kazemzadeh K, Vragniau C, Pelosi L, Pierrel F. 2020. Advances in bacterial pathways for the biosynthesis of ubiquinone. Biochim Biophys Acta Bioenerg 1861:148259.

15. Hajj Chehade M, Pelosi L, Fyfe CD, Loiseau L, Rascalou B, Brugiere S, Kazemzadeh K, Vo CD, Ciccone L, Aussel L, Coute Y, Fontecave M, Barras F, Lombard M, Pierrel F. 2019. A soluble metabolon synthesizes the isoprenoid lipid ubiquinone. Cell Chem Biol 26:482-492 e7.

16. Pelosi L, Vo CD, Abby SS, Loiseau L, Rascalou B, Hajj Chehade M, Faivre B, Gousse M, Chenal C, Touati N, Binet L, Cornu D, Fyfe CD, Fontecave M, Barras F, 
Lombard M, Pierrel F. 2019. Ubiquinone biosynthesis over the entire $\mathrm{O}_{2}$ range: characterization of a conserved $\mathrm{O}_{2}$-independent pathway. mBio 10:e01319-19.

17. Stenmark P, Grunler J, Mattsson J, Sindelar PJ, Nordlund P, Berthold DA. 2001. A new member of the family of di-iron carboxylate proteins. Coq7 (clk-1), a membranebound hydroxylase involved in ubiquinone biosynthesis. J Biol Chem 276:3329733300 .

18. Zhou L, Li M, Wang XY, Liu H, Sun S, Chen H, Poplawsky A, He YW. 2019. Biosynthesis of coenzyme $\mathrm{Q}$ in the Phytopathogen xanthomonas campestris via a

19. Pelosi L, Ducluzeau AL, Loiseau L, Barras F, Schneider D, Junier I, Pierrel F. 2016.

20. Poon WW, Davis DE, Ha HT, Jonassen T, Rather PN, Clarke CF. 2000. Identification of Escherichia coli ubiB, a gene required for the first monooxygenase step in

21. Aussel L, Loiseau L, Hajj Chehade M, Pocachard B, Fontecave M, Pierrel F, Barras F. 2014. ubiJ, a new gene required for aerobic growth and proliferation in macrophage, is involved in coenzyme Q biosynthesis in Escherichia coli and Salmonella enterica serovar Typhimurium. J Bacteriol 196:70-79.

22. Hajj Chehade M, Loiseau L, Lombard M, Pecqueur L, Ismail A, Smadja M, GolinelliPimpaneau B, Mellot-Draznieks C, Hamelin O, Aussel L, Kieffer-Jaquinod S, Labessan N, Barras F, Fontecave M, Pierrel F. 2013. ubiI, a new gene in Escherichia

23. Loiseau L, Fyfe C, Aussel L, Hajj Chehade M, Hernandez SB, Faivre B, Hamdane D, Mellot-Draznieks C, Rascalou B, Pelosi L, Velours C, Cornu D, Lombard M, coli coenzyme Q biosynthesis, is involved in aerobic C5-hydroxylation. J Biol Chem 288:20085-20092.

24. Thelaus J, Lundmark E, Lindgren P, Sjodin A, Forsman M. 2018. Galleria mellonella Casadesus J, Pierrel F, Fontecave M, Barras F. 2017. The UbiK protein is an accessory 
25. Aperis G, Fuchs BB, Anderson CA, Warner JE, Calderwood SB, Mylonakis E. 2007. Galleria mellonella as a model host to study infection by the Francisella tularensis live vaccine strain. Microbes Infect 9:729-734.

26. Propst CN, Pylypko SL, Blower RJ, Ahmad S, Mansoor M, van Hoek ML. 2016. Francisella philomiragia Infection and lethality in mammalian tissue culture cell models, Galleria mellonella, and BALB/c mice. Front Microbiol 7:696.

27. Brodmann M, Schnider S, Basler M. 2021. Type VI secretion system and its effectors PdpC, PdpD and OpiA contribute to Francisella virulence in Galleria mellonella larvae. Infect Immun 89:e0057920.

28. Tang Q, Feng M, Xia H, Zhao Y, Hou B, Ye J, Wu H, Zhang H. 2019. Differential quantitative proteomics reveals the functional difference of two yig $P$ locus products, UbiJ and EsrE. J Basic Microbiol 59:1125-1133.

29. Jiang HX, Wang J, Zhou L, Jin ZJ, Cao XQ, Liu H, Chen HF, He YW. 2019. Coenzyme Q biosynthesis in the biopesticide Shenqinmycin-producing Pseudomonas aeruginosa strain M18. J Ind Microbiol Biotechnol 46:1025-1038.

30. Nakahigashi K, Miyamoto K, Nishimura K, Inokuchi H. 1992. Isolation and characterization of a light-sensitive mutant of Escherichia coli K-12 with a mutation in a gene that is required for the biosynthesis of ubiquinone. J Bacteriol 174:73527359.

31. Zhou L, Wang JY, Wu J, Wang J, Poplawsky A, Lin S, Zhu B, Chang C, Zhou T, Zhang LH, He YW. 2013. The diffusible factor synthase XanB2 is a bifunctional chorismatase that links the shikimate pathway to ubiquinone and xanthomonadins biosynthetic pathways. Mol Microbiol 87:80-93.

32. Marshall SA, Payne KAP, Leys D. 2017. The UbiX-UbiD system: The biosynthesis and use of prenylated flavin (prFMN). Arch Biochem Biophys 632:209-221.

33. Ravcheev DA, Thiele I. 2016. Genomic analysis of the human gut microbiome suggests novel enzymes involved in quinone biosynthesis. Front Microbiol 7:128.

34. Degli Esposti M. 2017. A journey across genomes uncovers the origin of ubiquinone in cyanobacteria. Genome Biol Evol 9:3039-3053.

35. Sharma P, Teixeira de Mattos MJ, Hellingwerf KJ, Bekker M. 2012. On the function of the various quinone species in Escherichia coli. FEBS J 279:3364-3373.

36. Ziveri J, Barel M, Charbit A. 2017. Importance of metabolic adaptations in francisella pathogenesis. Front Cell Infect Microbiol 7:96. 
37. Dieppedale J, Gesbert G, Ramond E, Chhuon C, Dubail I, Dupuis M, Guerrera IC, Charbit A. 2013. Possible links between stress defense and the tricarboxylic acid (TCA) cycle in Francisella pathogenesis. Mol Cell Proteomics 12:2278-2292.

38. Chong A, Celli J. 2010. The Francisella intracellular life cycle: toward molecular mechanisms of intracellular survival and proliferation. Front Microbiol 1:138.

39. Brissac T, Ziveri J, Ramond E, Tros F, Kock S, Dupuis M, Brillet M, Barel M, Peyriga L, Cahoreau E, Charbit A. 2015. Gluconeogenesis, an essential metabolic pathway for pathogenic Francisella. Mol Microbiol 98:518-534.

40. Austin D, Larson TJ. 1991. Nucleotide sequence of the $g l p D$ gene encoding aerobic sn-glycerol 3-phosphate dehydrogenase of Escherichia coli K-12. J Bacteriol 173:101-107.

41. Soballe B, Poole RK. 2000. Ubiquinone limits oxidative stress in Escherichia coli. Microbiology (Reading) 146:787-796.

42. Kinkead LC, Allen LA. 2016. Multifaceted effects of Francisella tularensis on human neutrophil function and lifespan. Immunol Rev 273:266-281.

43. Steiner DJ, Furuya Y, Jordan MB, Metzger DW. 2017. Protective role for macrophages in respiratory Francisella tularensis Infection. Infect Immun 85:e006417.

44. Felix J, Siebert C, Ducassou JN, Nigou J, Garcia PS, Fraudeau A, Huard K, Mas C, Brochier-Armanet C, Coute Y, Gutsche I, Renesto P. 2021. Structural and functional analysis of the Francisella lysine decarboxylase as a key actor in oxidative stress resistance. Sci Rep 11:972.

45. Chamberlain RE. 1965. Evaluation of live tularemia vaccine prepared in a chemically defined medium. Appl Microbiol 13:232-235.

46. Datsenko KA, Wanner BL. 2000. One-step inactivation of chromosomal genes in Escherichia coli K-12 using PCR products. Proc Natl Acad Sci USA 97:6640-6645.

47. Baba T, Ara T, Hasegawa M, Takai Y, Okumura Y, Baba M, Datsenko KA, Tomita M, Wanner BL, Mori H. 2006. Construction of Escherichia coli K-12 in-frame, singlegene knockout mutants: the Keio collection. Mol Syst Biol 2:2006.0008. 
A

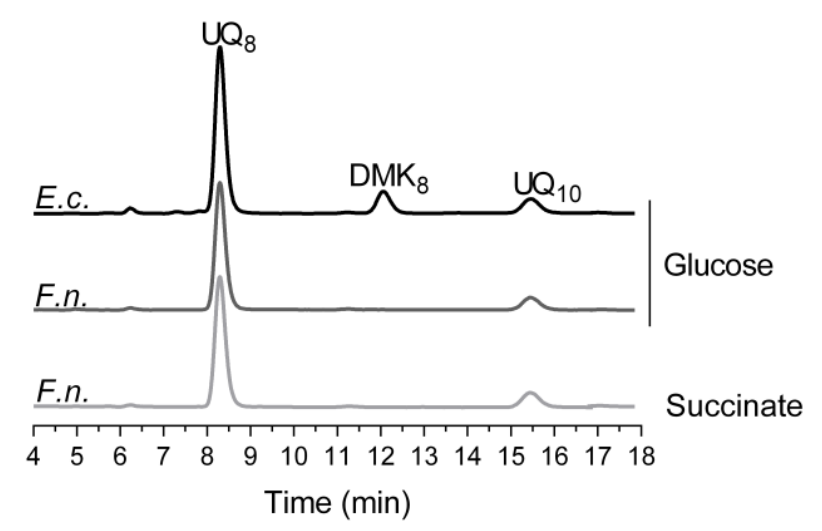

B

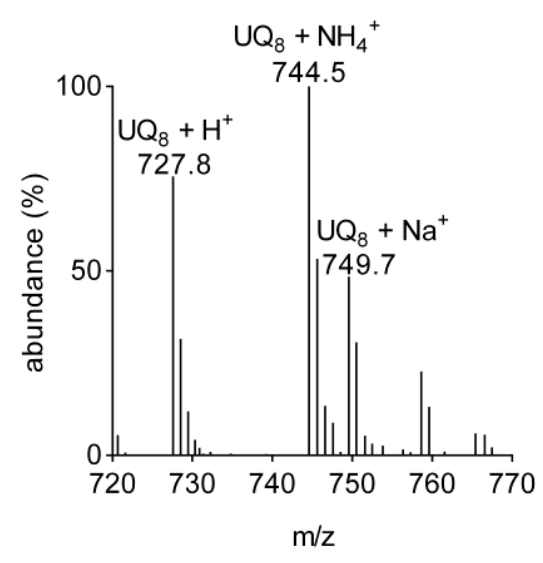

621 Figure 1. UQ $\mathbf{Q}_{\mathbf{8}}$ is the major quinone used by $\boldsymbol{F}$. novicida. (A) HPLC-ECD analysis of lipid 622 extracts from $1 \mathrm{mg}$ of E. coli MG1655 (E.c.) and F. novicida (F.n.) cells grown aerobically in 623 Chamberlain medium with $0.4 \%(\mathrm{w} / \mathrm{v})$ either glucose or succinate as the sole carbon source. 624 The chromatograms are representative of three independent experiments. The peaks 625 corresponding to $\mathrm{UQ}_{8}, \mathrm{DMK}_{8}$ and the $\mathrm{UQ}_{10}$ standard are indicated. (B) Mass spectrum of the 626 quinone eluting at $8.30 \mathrm{~min}$ from extracts of $F$. novicida grown in the Chamberlain media. $\mathrm{H}^{+}$, $627 \mathrm{NH}_{4}{ }^{+}$and $\mathrm{Na}^{+}$adducts of $\mathrm{UQ}_{8}$ are indicated. 
A

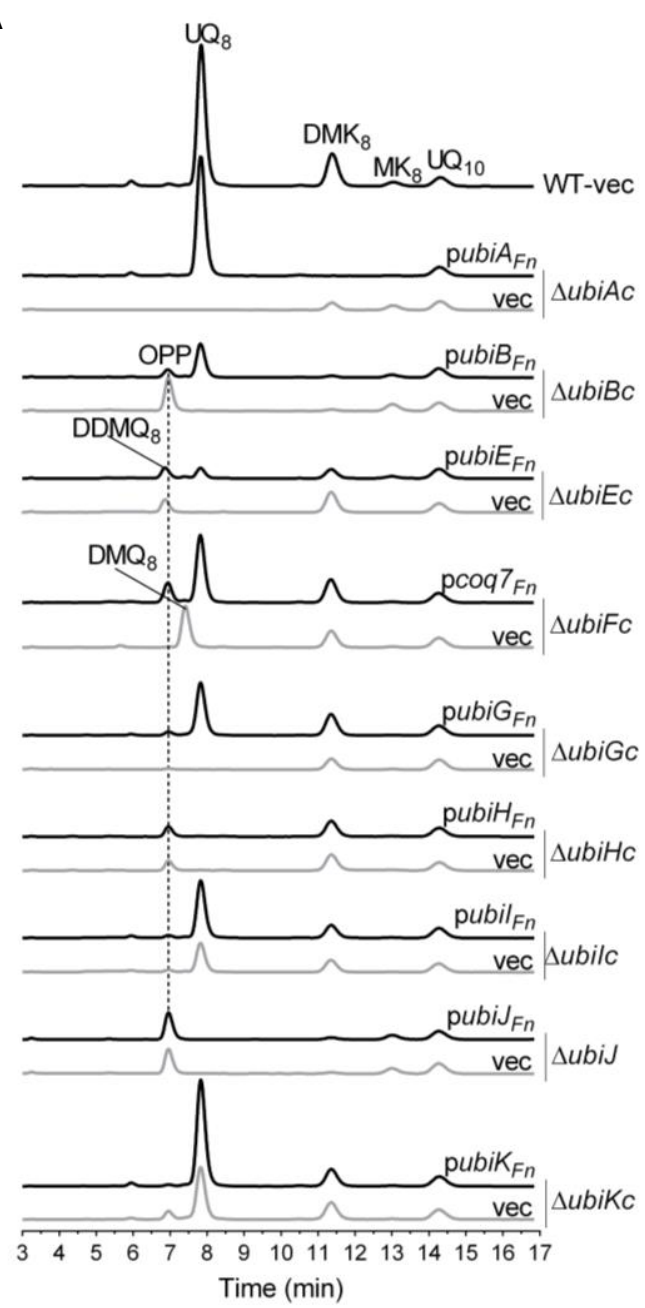

B

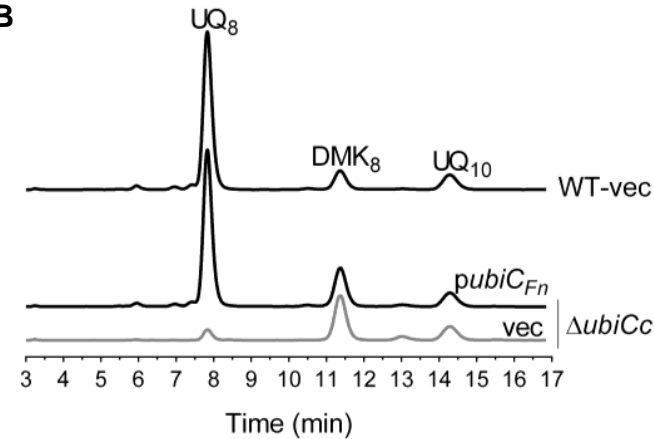

C

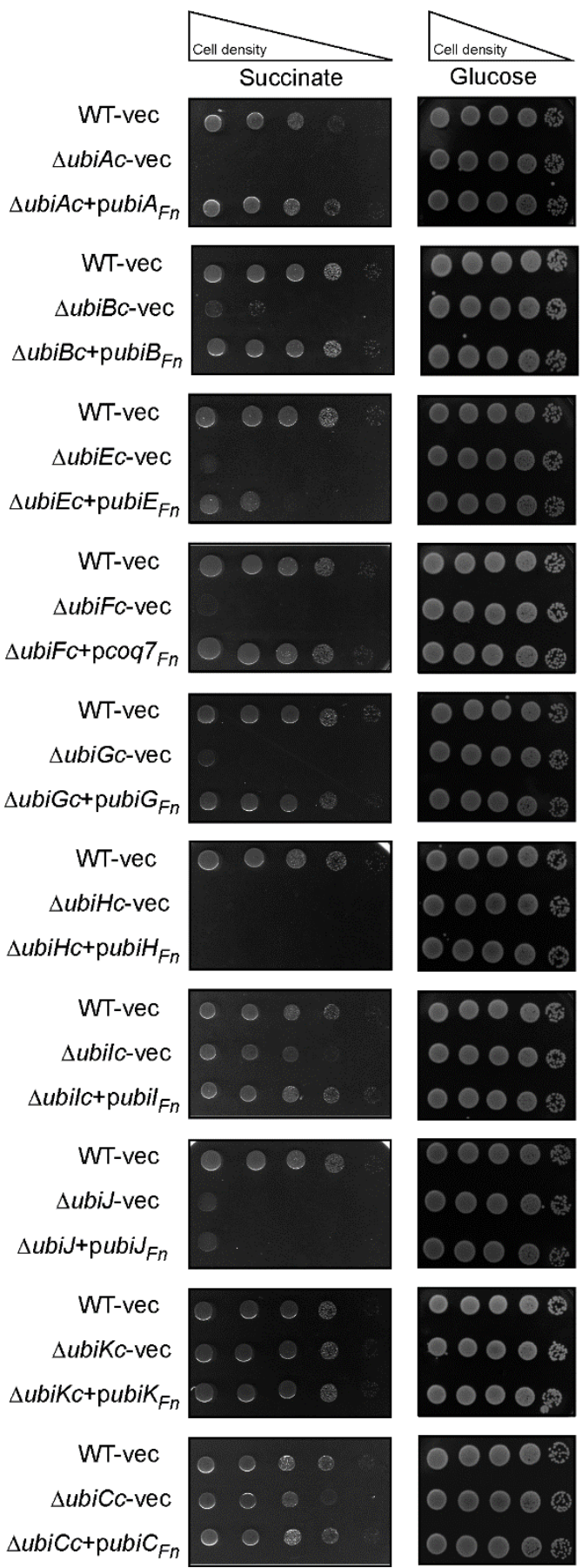

Figure 2. Complementation analysis of $E$. coli $\mathrm{UQ}_{8}$ biosynthesis mutants with the putative Ubi-proteins from $\boldsymbol{F}$. novicida. The $\Delta u b i$ E. coli mutant strains transformed with pTrc99a (vec) or pTrc99a encompassing the $u b i_{F n}$ genes were grown over night at $37^{\circ} \mathrm{C}$ in $\mathrm{LB}$ medium (A) or in M9 minimal medium (B) with $0.4 \%$ (w/v) glucose as the sole carbon source. Expression of the $\mathrm{Ubi}_{F n}$-proteins was induced by addition of IPTG to a final concentration of $100 \mu \mathrm{M}$. The $E$. coli wild-type strain MG1655 (WT) transformed with the pTrc99a empty vector was used as a control. HPLC-ECD analysis of lipid extracts from $1 \mathrm{mg}$ of cells. The chromatograms are representative of three independent experiments. The peaks corresponding to $\mathrm{OPP}, \mathrm{DDMQ}_{8}, \mathrm{DMQ}_{8}, \mathrm{UQ}_{8}, \mathrm{MK}_{8}, \mathrm{DMK}_{8}$ and the $\mathrm{UQ}_{10}$ standard are indicated. (C) Serial dilutions were spotted onto plates containing M9 minimal medium with $0.4 \%(w / v)$ either glucose or succinate as the sole carbon source and IPTG (100 $\mu \mathrm{M}$ final concentration). The plates were incubated overnight at $37^{\circ} \mathrm{C}$. 
A

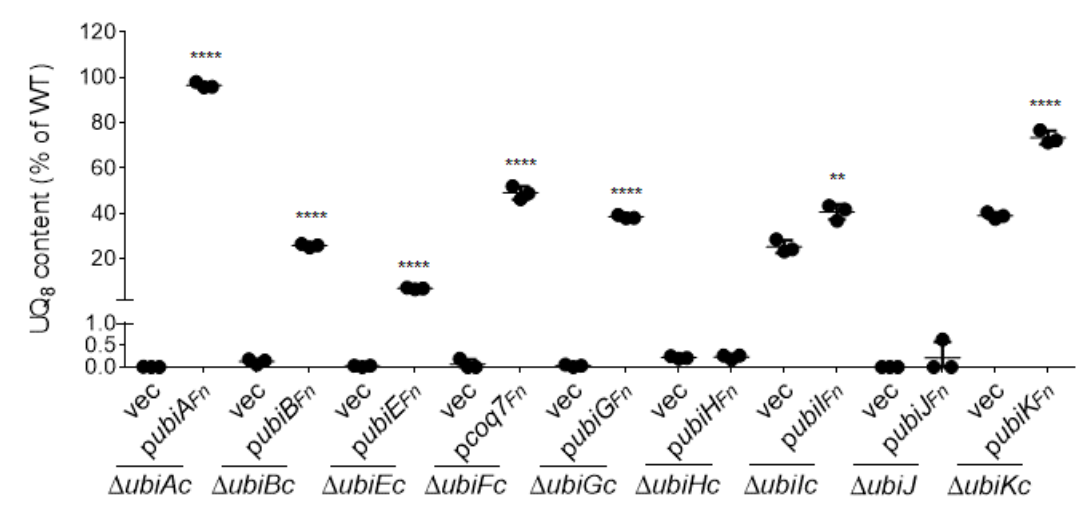

B

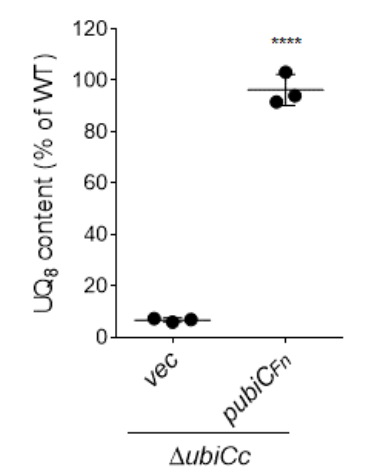

641 Figure 3. Quantification of cellular UQ8 contents of $\Delta u b i E$. coli mutant strains 642 expressing the $\mathbf{U b i}_{\boldsymbol{F n}}$-proteins. The $\Delta u b i$ E. coli mutant strains transformed with pTrc99a 643 (vec) or pTrc99a encompassing the $u b i_{F n}$-genes were grown over night at $37^{\circ} \mathrm{C}$ in LB medium 644 (A) or in M9 minimal medium (B) with $0.4 \%$ (w/v) glucose as the sole carbon source. 645 Expression of the $\mathrm{Ubi}_{F n}$-proteins was described in the legend of the Figure 2. Quantifications 646 are expressed as the percentages of the control value, which corresponds to the $\mathrm{UQ}_{8}$ content 647 of the wild-type strain $(\mathrm{n}=3)$. ****, $\mathrm{P}<0.0001$ and $* *, \mathrm{P}<0.005$ by unpaired Student's $\mathrm{t}$ 648 test. 
A

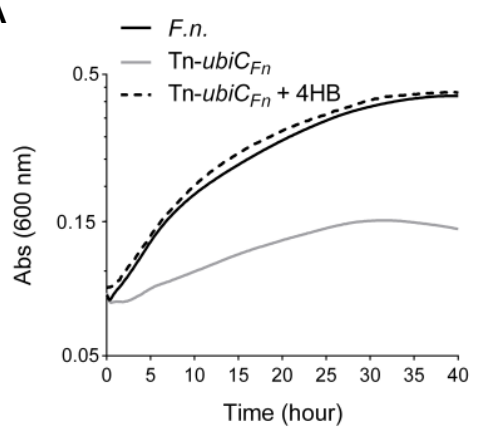

B

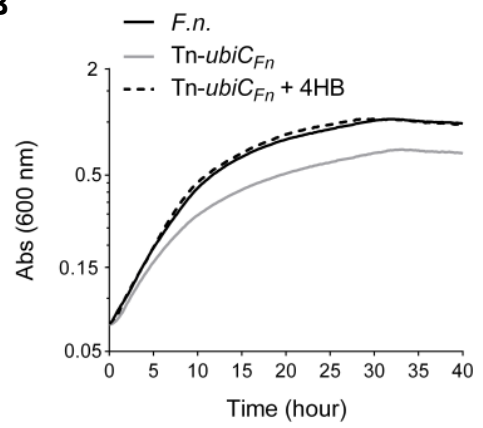

C

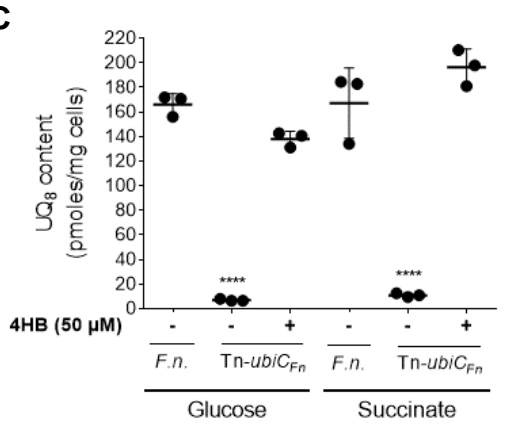

650 Figure 4. $\mathrm{UQ}_{8}$ is essential for the growth of $F$. novicida in respiratory medium. $F$. 651 novicida (F.n.) and transposon mutant of $u b i C_{F n}\left(\mathrm{Tn}-u b i C_{F n}\right)$ were grown aerobically in 652 Chamberlain medium with $0.4 \%$ (w/v) either succinate (A) or glucose (B) as the sole carbon 653 source. Growth (average of sixtiplicate growth curves) was followed as the change in the 654 absorbance at $600 \mathrm{~nm}$ in a Tecan plate reader. (C) Cellular UQ $\mathrm{U}_{8}$ contents were quantified for $655 F . n$. and Tn-ubi $C_{F n}$ according to the materials and methods section. 4HB was added to rescue 656 the growth and the $\mathrm{UQ}_{8}$ biosynthesis in $\mathrm{Tn}-u b i C_{F n}$. Quantifications are expressed as pmoles per mg of cells $(\mathrm{n}=3)$. $* * * *, \mathrm{P}<0.0001$ by unpaired Student's $\mathrm{t}$ test. 
A

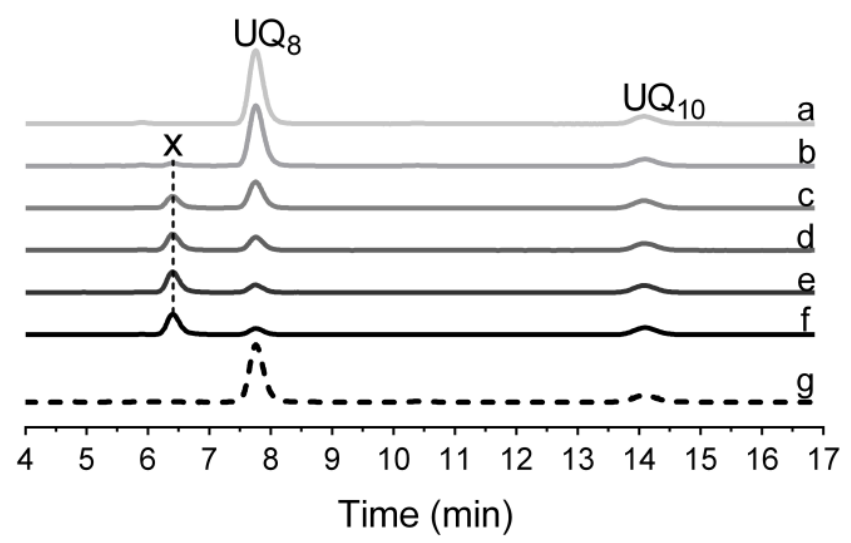

B

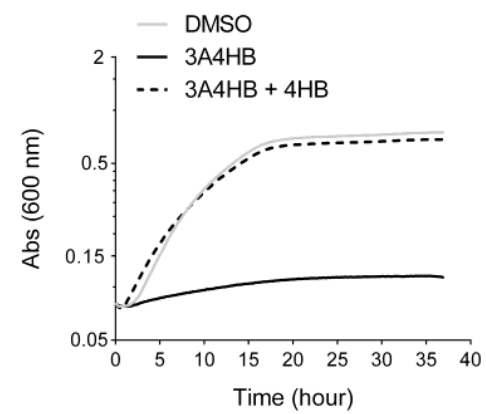

C

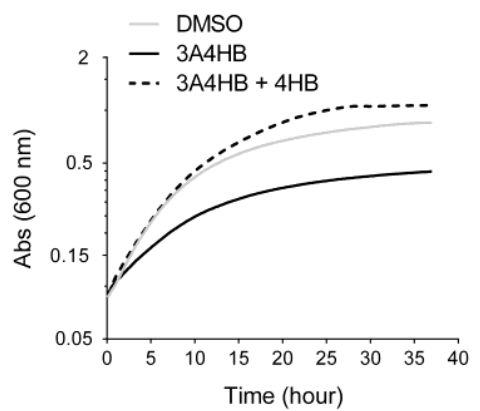

D

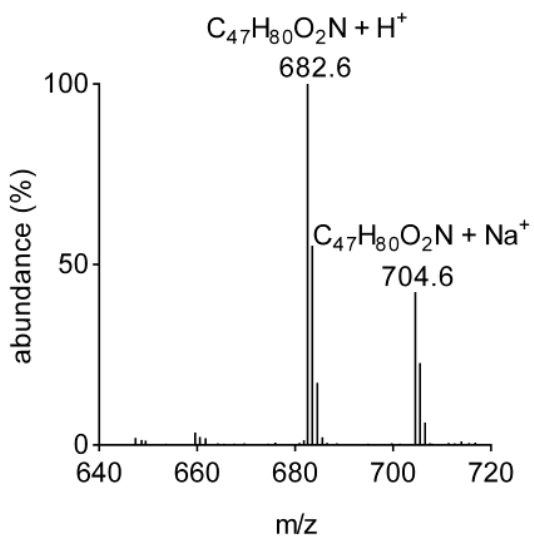

E<smiles>CC/C(C)=C/CC1=C(C)C(=O)C=C(N)C1=O</smiles>

659 Figure 5. Effect of 3A4HB on UQ8 biosynthesis and growth of $\boldsymbol{F}$. novicida. (A) HPLC660 ECD analysis of lipid extracts from $1 \mathrm{mg}$ of $F$. novicida cells grown aerobically in 661 Chamberlain medium with $0.4 \%(\mathrm{w} / \mathrm{v})$ glucose as the sole carbon source and in the presence 662 of different concentration of 3A4HB solubilized in DMSO $(\mathrm{a}=\mathrm{DMSO} ; \mathrm{b}=0.01 \mathrm{mM} ; \mathrm{c}=0.1$ $\mathrm{mM} ; \mathrm{d}=0.25 \mathrm{mM} ; \mathrm{e}=0.5 \mathrm{mM} ; \mathrm{f}=1 \mathrm{mM} ; \mathrm{g}=1 \mathrm{mM} 3 \mathrm{~A} 4 \mathrm{HB}+1 \mathrm{mM} 4 \mathrm{HB})$. The chromatograms are representative of three independent experiments. The peaks corresponding to $\mathrm{UQ}_{8}$ and the $\mathrm{UQ}_{10}$ standard, are indicated. Compound $\mathrm{X}$ eluting at $6.5 \mathrm{~min}$ is marked. Growth curves for $F$. novicida cultured under aerobic conditions in Chamberlain medium with $0.4 \%(\mathrm{w} / \mathrm{v})$ either succinate (B) or glucose (C) as the sole carbon source and in the presence of either DMSO (control), $1 \mathrm{mM} 3 \mathrm{~A} 4 \mathrm{HB}$ or $1 \mathrm{mM} 3 \mathrm{~A} 4 \mathrm{HB}+100 \mu \mathrm{M} 4 \mathrm{HB}$. The growth for each condition (average of sixtiplicate growth curves) was followed as the change in the absorbance at $600 \mathrm{~nm}$ in a Tecan plate reader. (D) Mass spectrum of compound X eluting from extracts of $F$. novicida grown in the Chamberlain media with $1 \mathrm{mM}$ of $3 \mathrm{~A} 4 \mathrm{HB} . \mathrm{H}^{+}$and $\mathrm{Na}^{+}$adducts corresponding to this molecule were indicated. (E) Proposed structure of compound $\mathrm{X}$ in its oxidized form. 
A

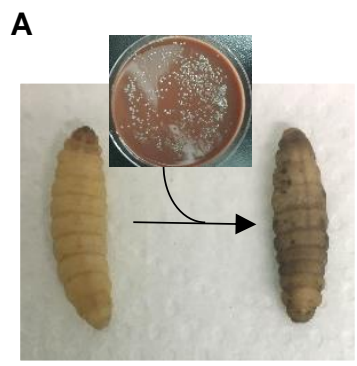

B

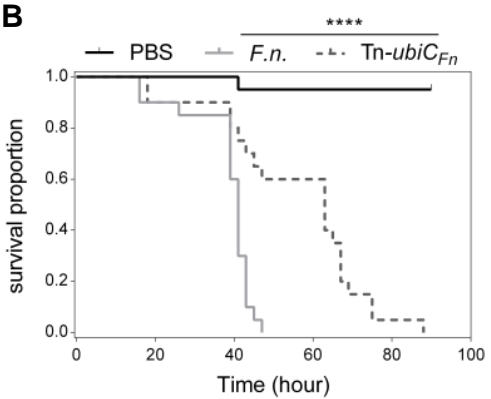

C

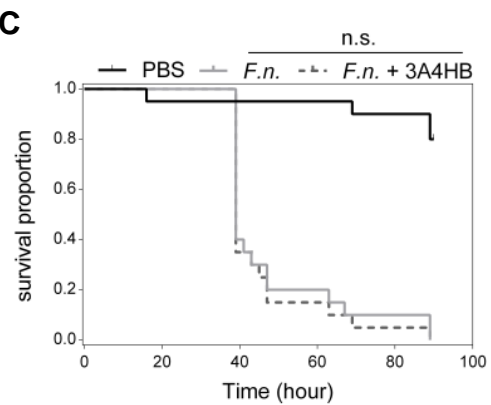

675

676

677

678

679

680

681

682

Figure 6: $\mathrm{UQ}_{8}$ contributes to the later steps of the infection of $F$. novicida in $G$. mellonella. (A) The larvae are turning grey/black when infected. (B) Survival curve of $G$. mellonella infected with either $F$. novicida $\left(F . n\right.$.) or the transposon mutant of $u b i C_{F n}(\mathrm{Tn}$ $\left.u b i C_{F n}\right)$. ****, $\mathrm{P}<0.0001$ by Log-rank (Mantel-Cox) test. (C) Survival curve of G. mellonella infected with $F$. novicida (F.n.) pre-treated or not with $3 \mathrm{~A} 4 \mathrm{HB}(1 \mathrm{mM}$ final concentration). Each group of $G$. mellonella $(\mathrm{n}=20)$ was injected with around $10^{6} \mathrm{CFU} /$ arva and PBS injection was used as control. n.s. (no significant), $\mathrm{P}=0.6670$ by Log-rank (Mantel-Cox) test. 


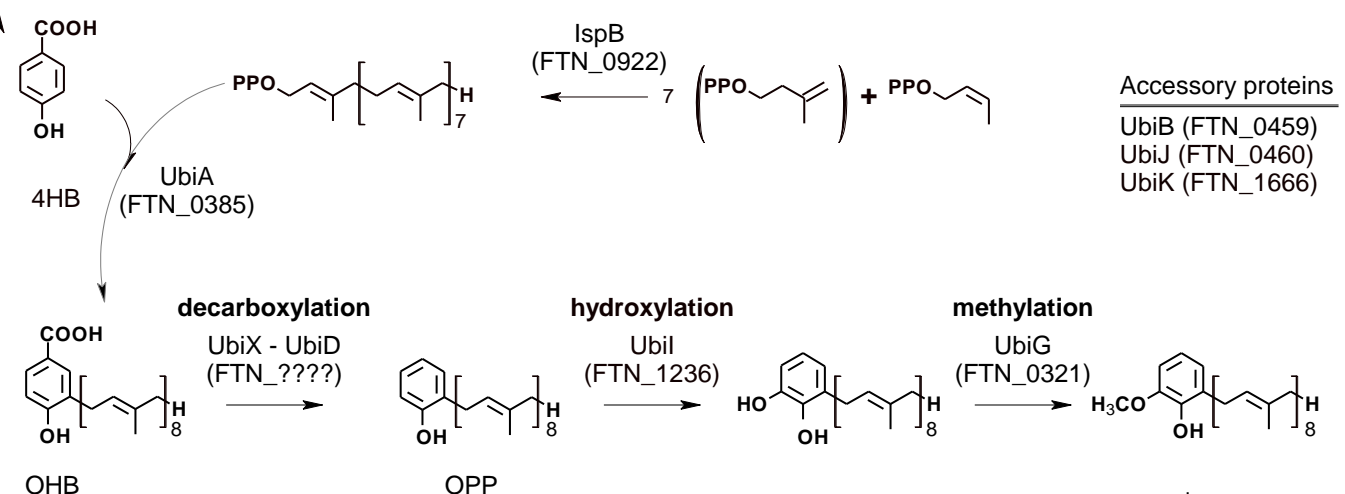

$\mathrm{OHB}$

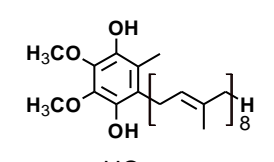

$\mathrm{UQ}_{8}$

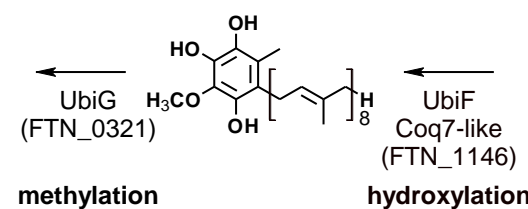

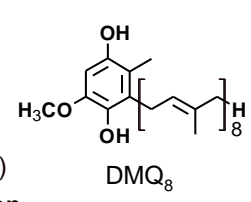

$\mathrm{DMQ}_{8}$

\section{Figure S1: Proposed $\mathrm{UQ}_{8}$ biosynthetic pathway in $F$. novicida deduced from the one} characterized in $\boldsymbol{E}$. coli. Corresponding protein IDs in $F$. novicida are indicated in parenthesis. There is no identified counterpart of UbiD and UbiX in F. novicida proteome. UbiF is only identified in E. coli and its functional homolog in F. novicida is a Coq7 hydroxylase. Abbreviations used are 4HB, 4-hydroxybenzoic acid; OHB, 3-octaprenyl-4hydroxybenzoic acid; OPP, octaprenylphenol; $\mathrm{DMQ}_{8}, \mathrm{C6}$-demethoxy-ubiquinone 8; $\mathrm{DDMQ}_{8}$, C1-demethyl-C6-demethoxy-ubiquinone 8; $\mathrm{UQ}_{8}$, ubiquinone 8. 


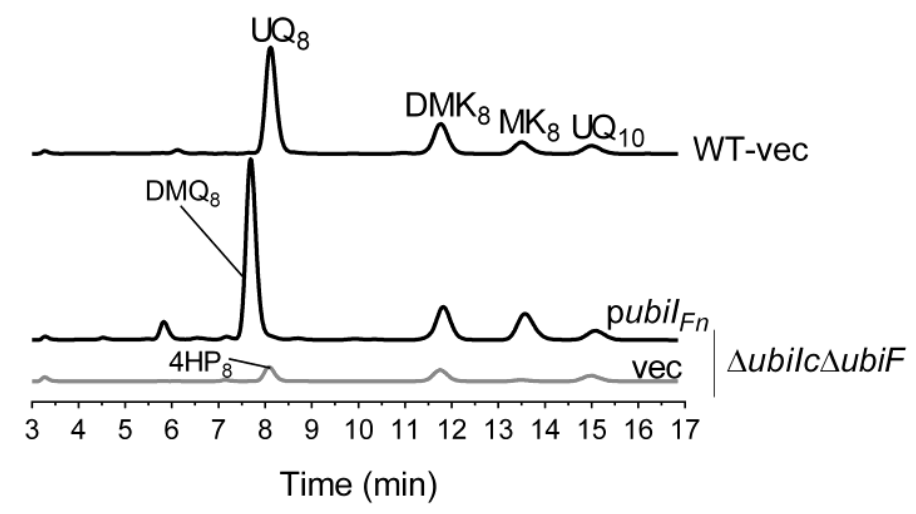

692 Figure S2. Complementation analysis of $E$. coli $\Delta u b i I c \Delta u b i F$ mutant with UbiI $_{F n}$. The $E$.

693 coli $\Delta u b i I c \Delta u b i F$ mutant strain transformed with pTrc99a (vec) or pTrc99a encompassing the $694 u b i I_{F n}$ gene were grown over night at $37^{\circ} \mathrm{C}$ in LB medium. Expression of the UbiI $F n$ protein 695 was induced by addition of IPTG to a final concentration of $100 \mu \mathrm{M}$. The $E$. coli wild-type 696 strain MG1655 (WT) transformed with the pTrc99a empty vector was used as a control. 697 HPLC-ECD analysis of lipid extracts from $1 \mathrm{mg}$ amounts of cells. The chromatograms are 698 representative of three independent experiments. The peaks corresponding to $4 \mathrm{HP}_{8}, 3$ 699 octaprenyl-4-hydroxyphenol; $\mathrm{DMQ}_{8}, \mathrm{C6}$-demethoxy-ubiquinone; $\mathrm{UQ}_{8}$, ubiquinone 8; $\mathrm{MK}_{8}$, 700 menaquinone 8 ; $\mathrm{DMK}_{8}$, demethylmenaquinone 8 and $\mathrm{UQ}_{10}$, ubiquinone 10 as a standard, are 701 indicated. 
A<smiles>O=C(O)c1ccc(O)cc1</smiles>

4HB<smiles>Nc1ccc(C(=O)O)cc1</smiles>

pABA<smiles>COc1cc(N)ccc1C(=O)O</smiles>

pA2MBA<smiles>COc1cc(C(=O)O)ccc1N</smiles>

pA3MBA<smiles>Nc1cc(C(=O)O)ccc1O</smiles>

3A4HB
B

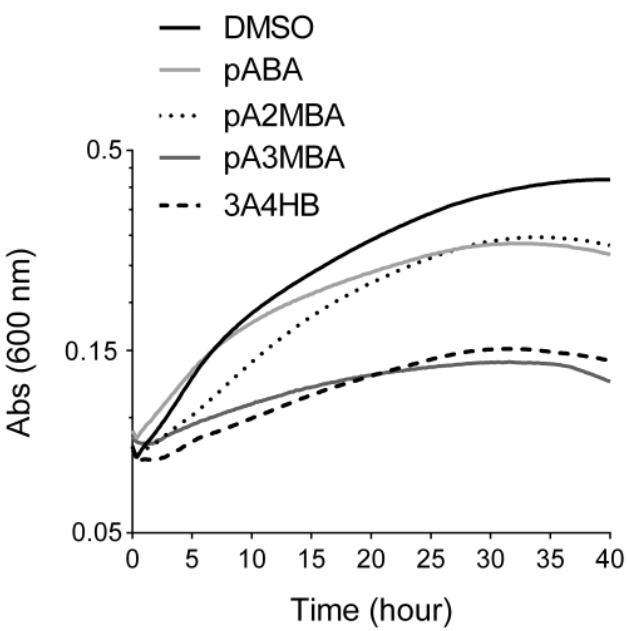

C

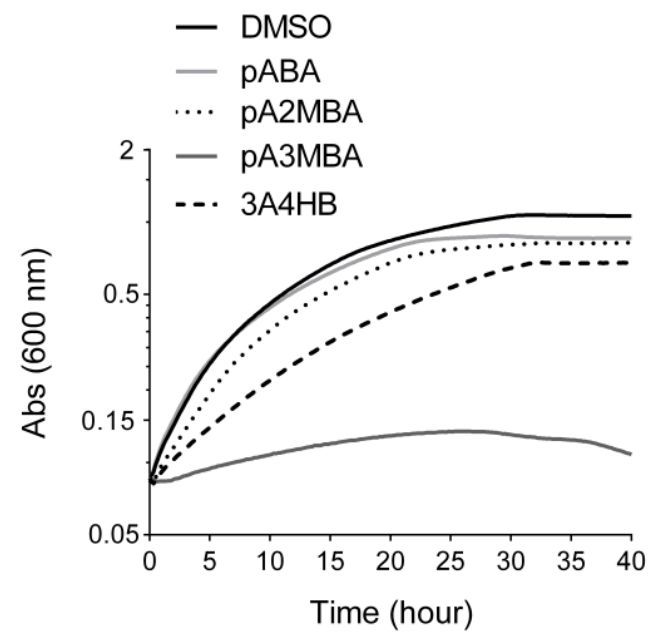

Figure S3. Effect of derivative compounds of $4 \mathrm{HB}$ on $\boldsymbol{F}$. novicida growth. (A) Chemical structures of the compounds tested. Growth curves for $F$. novicida cultivated under aerobic conditions in Chamberlain medium with $0.4 \%$ (w/v) succinate (B) or glucose (C) as the sole carbon source and in the presence of $1 \mathrm{mM}$ of the various $4 \mathrm{HB}$ analogues solubilized in DMSO (average of sixtiplicate growth curves). DMSO was used as a control. The growth for each condition was followed as the change in the absorbance at $600 \mathrm{~nm}$ in a Tecan plate reader. More details of growth experiments are found in Materials and methods section. pABA, 4-amino-benzoic acid; pA2MBA, 4-amino-2-methoxy-benzoic acid; pA3MBA, 4amino-3-methoxy-benzoic acid and 3A4HB, 3-amino-4-hydroxybenzoic acid. 
Table S1: Bioinformatics analysis of the proteins required for UQ biosynthesis in $E$. coli MG1655, in F. novicida U112 and in F.

tularensis ssp tularensis Schu S4. ${ }^{(1)}$ Deduced from (1). ${ }^{(2)}$ From https://ecocyc.org/. ${ }^{(3)}$ From (2).

\begin{tabular}{|c|c|c|c|c|c|c|c|c|c|}
\hline \multicolumn{4}{|c|}{$\begin{array}{c}\text { E. coli } \\
\text { MG1655 }\end{array}$} & \multicolumn{3}{|c|}{$\begin{array}{l}\text { F. novicida } \\
\text { U112 }\end{array}$} & \multicolumn{2}{|c|}{$\begin{array}{l}\text { F. tularensis ssp } \\
\text { tularensis } \text { Schu S4 }\end{array}$} & \\
\hline \multirow{2}{*}{ Protein } & \multirow{2}{*}{$\begin{array}{l}\text { Size } \\
(\text { aa) }\end{array}$} & \multicolumn{2}{|c|}{$\begin{array}{c}\text { Need in UQ } \\
\text { biosynthesis }{ }^{(1)}\end{array}$} & \multirow{2}{*}{ Protein ID } & \multirow{2}{*}{$\begin{array}{l}\text { Size } \\
(\text { aa) }\end{array}$} & \multirow{2}{*}{$\begin{array}{l}\% \text { of } \\
\text { identity }\end{array}$} & \multirow{2}{*}{ Protein ID } & \multirow{2}{*}{$\begin{array}{l}\% \text { of } \\
\text { identity }\end{array}$} & \multirow{2}{*}{ Functions in $E$. coli $^{(2)}$ or specific to $F$. novicida } \\
\hline & & $+\mathrm{O}_{2}$ & $-\mathrm{O}_{2}$ & & & & & & \\
\hline IspB & 323 & \multicolumn{2}{|c|}{ Yes } & FTN_0922 & 326 & 48 & FTT_1044c & 48 & all-trans-octaprenyl-diphosphate synthase \\
\hline UbiA & 290 & \multicolumn{2}{|c|}{ Yes } & FTN_0385 & 285 & 42 & FTT_0856c & 41 & 4-HB octaprenyl transferase \\
\hline UbiB & 546 & \multicolumn{2}{|c|}{ Yes } & FTN_0459 & 553 & 47 & FTT_1298 & 48 & Essential to UQ biosynthesis \\
\hline UbiC & 165 & \multicolumn{2}{|c|}{ Yes } & FTN_0386 & 169 & 28 & FTT_0857c & 27 & Chorismate pyruvate lyase \\
\hline UbiD & 497 & \multicolumn{2}{|c|}{ Yes } & \multicolumn{5}{|c|}{ No homolog } & 3-octaprenyl 4-HB decarboxylase \\
\hline UbiE & 251 & \multicolumn{2}{|c|}{ Yes } & FTN_0461 & 251 & 70 & FTT_1296 & 70 & 2-octaprenyl-6-methoxy-1,4-benzoquinone methylase \\
\hline UbiF & 391 & \multicolumn{2}{|c|}{ Yes } & \multicolumn{5}{|c|}{ No homolog } & 2-octaprenyl-3-methyl-6-methoxy-1,4-benzoquinol hydroxylase \\
\hline Coq7 & \multicolumn{3}{|c|}{ No homolog } & \multicolumn{3}{|c|}{ FTN_1146 } & \multicolumn{2}{|c|}{ FTT_0537 } & 2-nonaprenyl-3-methyl-6-methoxy-1,4-benzoquinol hydroxylase ${ }^{(3)}$ \\
\hline UbiG & 240 & \multicolumn{2}{|c|}{ Yes } & FTN_0321 & 232 & 48 & FTT_1590c & 48 & $\begin{array}{l}\text { 3-demethylubiquinone-8 3-O-methyl transferase } \\
\text { 2-octaprenyl-6-hydroxyphenol methylase }\end{array}$ \\
\hline $\mathrm{UbiH}$ & 392 & Yes & No & FTN_1237 & 404 & 27 & FTT_1218c & 27 & 2-octaprenyl-6-methoxyphenol hydroxylase \\
\hline UbiI & 400 & Yes & No & FTN_1236 & 412 & 37 & FTT_1217c & 38 & 2-octaprenylphenol hydroxylase \\
\hline UbiJ & 201 & Yes & No & FTN_0460 & 196 & 21 & FTT_1297 & 21 & Essential to UQ biosynthesis-SCP2 domain \\
\hline UbiK & 96 & Yes & No & FTN_1666 & 65 & 44 & FTT_0045 & 44 & Ubiquinone biosynthesis accessory factor \\
\hline UbiT & 174 & No & Yes & \multirow{4}{*}{\multicolumn{5}{|c|}{ No homolog }} & Essential to UQ biosynthesis-SCP2 domain \\
\hline UbiU & 331 & No & Yes & & & & & & Essential to UQ biosynthesis \\
\hline UbiV & 292 & No & Yes & & & & & & Essential to UQ biosynthesis \\
\hline UbiX & 189 & \multicolumn{2}{|c|}{ Yes } & & & & & & Flavin prenyltransferase - UbiD partner protein \\
\hline
\end{tabular}

1. Pelosi L, Vo CD, Abby SS, Loiseau L, Rascalou B, Hajj Chehade M, Faivre B, Gousse M, Chenal C, Touati N, Binet L, Cornu D, Fyfe CD, Fontecave M, Barras F, Lombard M, Pierrel F. 2019. Ubiquinone biosynthesis over the entire $\mathrm{O}_{2}$ range: characterization of a conserved $\mathrm{O}_{2}$-independent pathway. $m$ Bio 10:e01319-19.

2. Stenmark P, Grunler J, Mattsson J, Sindelar PJ, Nordlund P, Berthold DA. 2001. A new member of the family of di-iron carboxylate proteins. Coq7 (clk1), a membrane-bound hydroxylase involved in ubiquinone biosynthesis. J Biol Chem 276:33297-33300. 
bioRxiv preprint doi: https://doi.org/10.1101/2021.08.03.455006; this version posted August 5, 2021. The copyright holder for this preprint (which was not certified by peer review) is the author/funder. All rights reserved. No reuse allowed without permission.

\section{Ubiquinone and Francisella pathogenicity}

721 Table S2: Bacterial strains and plasmids used in this work.

\begin{tabular}{lll}
\hline $\begin{array}{l}\text { Strains or } \\
\text { plasmids }\end{array}$ & Relevant genotypes and properties & $\begin{array}{l}\text { References or } \\
\text { sources }\end{array}$ \\
Strains & & \\
\hline F. novicida $\mathrm{U} 112$ & Wild-type strain & CNR, Grenoble, \\
France
\end{tabular}

\begin{tabular}{ll}
\hline Plasmids & \\
pTrc99a & vector for inducible gene expression $\left(\mathrm{Amp}^{\mathrm{R}}\right)$ \\
p $u b i A_{F n}$ & pTrc99a carrying $u b i A_{F n}$ \\
p $u b i B_{F n}$ & pTrc99a carrying $u b i B_{F n}$ \\
p $u b i C_{F n}$ & pTrc99a carrying $u b i C_{F n}$ \\
p $u b i E_{F n}$ & pTrc99a carrying $u b i E_{F n}$ \\
p $u b i G_{F n}$ & pTrc99a carrying $u b i G_{F n}$ \\
p $u b i H_{F n}$ & pTrc99a carrying $u b i H_{F n}$ \\
p $u b i I_{F n}$ & pTrc99a carrying $u b i I_{F n}$ \\
pubiJ & pTrc99a carrying $u b i J_{F n}$ \\
p $u b i K_{F n}$ & pTrc99a carrying $u b i K_{F n}$ \\
p $c o q 7_{F n}$ & pTrc99a carrying $\operatorname{coq} 7_{F n}$ \\
\hline
\end{tabular}

(6)

This work

This work

This work

This work

This work

This work

This work

This work

This work

This work comprehensive transposon mutant library of Proc Natl Acad Sci U S A 104:1009-1014.

2. Hajj Chehade M, Loiseau L, Lombard M, Pecqueur L, Ismail A, Smadja M, GolinelliPimpaneau B, Mellot-Draznieks C, Hamelin O, Aussel L, Kieffer-Jaquinod S, Labessan N, 
bioRxiv preprint doi: https://doi.org/10.1101/2021.08.03.455006; this version posted August 5, 2021. The copyright holder for this preprint (which was not certified by peer review) is the author/funder. All rights reserved. No reuse allowed without permission.

\section{Ubiquinone and Francisella pathogenicity}

Barras F, Fontecave M, Pierrel F. 2013. ubiI, a new gene in Escherichia coli coenzyme Q biosynthesis, is involved in aerobic C5-hydroxylation. J Biol Chem 288:20085-20092.

3. Loiseau L, Fyfe C, Aussel L, Hajj Chehade M, Hernandez SB, Faivre B, Hamdane D, MellotDraznieks C, Rascalou B, Pelosi L, Velours C, Cornu D, Lombard M, Casadesus J, Pierrel F, Fontecave M, Barras F. 2017. The UbiK protein is an accessory factor necessary for bacterial ubiquinone (UQ) biosynthesis and forms a complex with the UQ biogenesis factor UbiJ. $J$ Biol Chem 292:11937-11950.

4. Pelosi L, Ducluzeau AL, Loiseau L, Barras F, Schneider D, Junier I, Pierrel F. 2016. Evolution of ubiquinone biosynthesis: multiple proteobacterial enzymes with various regioselectivities to catalyze three contiguous aromatic hydroxylation reactions. mSystems 1:e00091-16.

5. Aussel L, Loiseau L, Hajj Chehade M, Pocachard B, Fontecave M, Pierrel F, Barras F. 2014. $u b i J$, a new gene required for aerobic growth and proliferation in macrophage, is involved in coenzyme Q biosynthesis in Escherichia coli and Salmonella enterica serovar Typhimurium. $J$ Bacteriol 196:70-79.

6. Amann E, Ochs B, Abel KJ. 1988. Tightly regulated tac promoter vectors useful for the expression of unfused and fused proteins in Escherichia coli. Gene 69:301-315. 
744 Table S3: Primers used in this work. Restriction sites are underlined.

\begin{tabular}{|c|c|c|}
\hline Name & Sequence $\left(5^{\prime} \rightarrow 3^{\prime}\right)$ & Purpose \\
\hline Fw-ubiA $A_{F n}$ & TTTTGAATTCATGAATAAACAACAACTAAAAGCATATTTC & \multirow{2}{*}{$\begin{array}{l}\text { Cloning } \\
u b i A_{F n}\end{array}$} \\
\hline Rc-ubiA $_{\mathrm{Fn}}$ & TTTTGGATCCTCATAAAAATTCCTTGATTATATATTGTATC & \\
\hline Fw-ubiB ${ }_{\mathrm{Fn}}$ & TTTTGAATTCATGATTAAAAAATTTCTAAGGCTTATCTAC & \multirow{2}{*}{$\begin{array}{l}\text { Cloning } \\
\text { ubiB }_{F n}\end{array}$} \\
\hline Rc-ubiB $\mathrm{Fn}$ & TTTTGGATCCTTATTTCTCCTTTTTAAAACTATAGTAAATC & \\
\hline $\mathrm{Fw}_{\mathrm{W}} \mathrm{ubiC} \mathrm{C}_{\mathrm{Fn}}$ & TTTTGAATTCATGAATGTTATTAACAAAGAAAATCTAAC & \multirow{2}{*}{$\begin{array}{l}\text { Cloning } \\
u b i C_{F n}\end{array}$} \\
\hline Rc-ubiC ${ }_{\mathrm{Fn}}$ & TTTTGGATCCTCAGTATTGCTCGGGTAAAACTAG & \\
\hline Fw-ubiE $E_{\mathrm{Fn}}$ & TTTTGGATCCATGTCTAAAGAAAATAAAACAACAGATTTTG & \multirow{2}{*}{$\begin{array}{l}\text { Cloning } \\
u b i E_{F n}\end{array}$} \\
\hline Rc-ubiE $E_{\mathrm{Fn}}$ & TTTTAAGCTTTTAATATTTATATCCAATATGTAATGCTAC & \\
\hline Fw-ubiG ${ }_{\mathrm{Fn}}$ & TTTTGAATTCATGATTAATATAGATAACAATGAAGTTG & \multirow{2}{*}{$\begin{array}{l}\text { Cloning } \\
\text { ubiG }_{F n}\end{array}$} \\
\hline Rc-ubiG ${ }_{F n}$ & TTTTGGATCCTTATACCTTTCTAAAAGCTATAATATAATTAAC & \\
\hline Fw-ubiH ${ }_{\mathrm{Fn}}$ & TTTTGAATTCATGAATGCTAAATATGATGTGGCAATTG & \multirow{2}{*}{$\begin{array}{l}\text { Cloning } \\
\text { ubiH } F n\end{array}$} \\
\hline Rc-ubiH $_{\mathrm{Fn}}$ & TTTTGGATCCCTAACGCTCATCCTCGACCAC & \\
\hline $\mathrm{FW}_{\mathrm{W}} \mathrm{ubiI_{ \textrm {Fn } }}$ & TTTTGAATTCATGAGCGTTAGAAATATTCAAAAAGATGCTG & \multirow{2}{*}{$\begin{array}{l}\text { Cloning } \\
\text { ubiI } F n\end{array}$} \\
\hline Rc-ubiI $I_{\mathrm{Fn}}$ & TTTTGGATCCTTATAAAGCTTGCTTCACAACTATACTTTTAAC & \\
\hline FW-ubiJ $_{\mathrm{Fn}}$ & TTTTGAATTCATGCTAAAACTAGTAAACACAGCC & \multirow{2}{*}{$\begin{array}{l}\text { Cloning } \\
\text { ubiJ }_{F n}\end{array}$} \\
\hline Rc-ubiJ $_{\mathrm{Fn}}$ & TTTTGGATCCTTAATCATTGTTTAAGCCTTCTAATAATTTTAG & \\
\hline Fw-ubiK ${ }_{\mathrm{Fn}}$ & TTTTGGATCCATGAAGGAAATTCTAGGACCG & \multirow{2}{*}{$\begin{array}{l}\text { Cloning } \\
u b i K_{F n}\end{array}$} \\
\hline Rc-ubiK $_{\mathrm{Fn}}$ & TTTTAAGCTTTTATTTTTTCTCAGCTAAAAGCTTATC & \\
\hline FW- $\operatorname{coq} 7_{\mathrm{Fn}}$ & TTTTGAATTCATGAGAAAGTTATCATTTTTAGATAGG & \multirow{2}{*}{$\begin{array}{l}\text { Cloning } \\
\operatorname{coq} 7_{F n}\end{array}$} \\
\hline Rc-coq 7 Fn & TTTTGGATCCTCAAAATCTATATGCAAGAGTAGTC & \\
\hline 5'-wannerubiA & $\begin{array}{l}\text { TTTTTACCGGCGTCACCGTTGTACTAAGAGGAAAAAAATCATATG } \\
\text { AATATCCTCCTTA }\end{array}$ & \multirow{2}{*}{$\begin{array}{l}\text { Inactivation } \\
\text { of } u b i A\end{array}$} \\
\hline 3'-wannerubiA & $\begin{array}{l}\text { CAGAAGAAAGCCGGATGATCATCCGGCTTTTTTACATCAGTGTAG } \\
\text { GCTGGAGCTGCTTC }\end{array}$ & \\
\hline 5'-wannerubiJ & $\begin{array}{l}\text { CGCTGCATCGTGGTTATAAGTTCTGACAGGAGACCGGAACATAT } \\
\text { GAATATCCTCCTTA }\end{array}$ & \multirow{2}{*}{$\begin{array}{l}\text { Inactivation } \\
\text { of } u b i J\end{array}$} \\
\hline 3'-wannerubiJ & $\begin{array}{l}\text { CGTACTTCACCTGGCGTCATTTAGCCTCCAGTTTTTCCAGCCGTTT } \\
\text { GTGTAGGCTGGAGCTGCTTC }\end{array}$ & \\
\hline 5'-xbaIubiB & TCGACTCTAGAGATGACGCCAGGTGAAGTACGGCGC & \multirow{2}{*}{$\begin{array}{l}\text { Inactivation } \\
\text { of } u b i B\end{array}$} \\
\hline 3'-xbaIubiB & CTTCCGTCTAGATCAGCGTGTTTTGCGCCAACCGACAAACCAGG & \\
\hline
\end{tabular}

